\title{
ERROR ANALYSIS OF TREFFTZ-DISCONTINUOUS GALERKIN METHODS FOR THE TIME-HARMONIC MAXWELL EQUATIONS
}

\author{
RALF HIPTMAIR, ANDREA MOIOLA, AND ILARIA PERUGIA
}

\begin{abstract}
In this paper, we extend to the time-harmonic Maxwell equations the $p$-version analysis technique developed in [R. Hiptmair, A. Moiola and I. Perugia, Plane wave discontinuous Galerkin methods for the $2 D$ Helmholtz equation: analysis of the p-version, SIAM J. Numer. Anal., 49 (2011), 264-284] for Trefftz-discontinuous Galerkin approximations of the Helmholtz problem. While error estimates in a mesh-skeleton norm are derived parallel to the Helmholtz case, the derivation of estimates in a mesh-independent norm requires new twists in the duality argument. The particular case where the local Trefftz approximation spaces are built of vector-valued plane wave functions is considered, and convergence rates are derived.
\end{abstract}

\section{INTRODUCTION}

Non-polynomial finite element methods for time-harmonic wave problems have been designed in the last years in order to reduce the computational cost, with respect to more classical polynomial-based methods. Examples of such methods are the partition of unity finite element method of Babuška and Melenk [7, the discontinuous enrichment method [4, 46, the variational theory of complex rays (VTCR) [4], and the ultra weak variational formulation (UWVF) by Cessenat and Després [18. All these methods are of Trefftz type, namely, they are based on approximation spaces made of functions which are (locally) solutions to the considered PDE. We concentrate, in particular, on the UWVF, which has recently seen rapid algorithmic development and extensions; see [15, 23, 24, 29, 34, 35, 36, and we would like to analyze its application to the time-harmonic Maxwell equations, considering general Trefftz approximation spaces.

Since the UWVF can be regarded as a discontinuous Galerkin (DG) method with Trefftz basis functions (see [15, 22, 24]), we briefly review some literature on standard (i.e., polynomial-based) DG methods for the time-harmonic Maxwell equations. Some of them are based on the primal curl-curl formulation of the problem, neglecting the divergence-free condition. For consistent DG-discretizations, these methods are spurious-free (see [16, Sec. 6], 21, 27, 31, 48]). Other DG methods are based on "regularized" primal curl-curl formulations, with penalization of the divergence-free constraint. With constant weights in the penalty term, the divergence is controlled, but these methods are haunted by so-called spurious solutions in the case of strongly singular problems; see [33, 42. This is avoided by using

Received by the editor February 21, 2011 and, in revised form, September 3, 2011.

2010 Mathematics Subject Classification. Primary 65N15, 65N30, 35Q61.

Key words and phrases. Time-harmonic Maxwell's equation, discontinuous Galerkin methods, Trefftz methods, $p$-version error analysis, duality estimates, plane waves.

(C)2012 American Mathematical Society Reverts to public domain 28 years from publication 
weighted regularized formulations, with penalty weights depending on the distance from singularities; see [10, 11] and their references. Alternative approaches to control the divergence of the numerical solutions are based on mixed-DG formulations, see 32,43 .

In this paper, we consider a Trefftz-DG approximation to the homogeneous timeharmonic Maxwell equations with impedance boundary conditions. For previous work on the UWVF for Maxwell, we refer to [8, 17, 19, 34]; for different Trefftz-based approaches, we mention [20, 47]. Taking a cue from the UWVF and following [34, we study a class of Trefftz methods that rely on a DG formulation of the electric field-based Maxwell problem, where the divergence-free constraint is not imposed; the discrete solutions will be elementwise divergence-free, but not globally. Our analysis applies to all these methods, independently of the choice of the particular Trefftz approximation space.

Our focus here is on the theoretical analysis of the $p$-version of the methods, which is immune to the pollution effect, an advantage also shared by spectral polynomial approximations; see [1, 2, 3]. The analysis framework presented in this paper is borrowed from [29. The first step consists of identifying a mesh skeleton norm on the Trefftz function space for which the bilinear form defining the method is coercive. This allows us to prove well-posedness and error estimates in this norm.

In order to derive error estimates in a mesh-independent norm, we use a duality argument introduced in [41] and used in [15, 29] for the Helmholtz problem. In order to extend this argument to the Maxwell equations, stability and regularity results for the Maxwell equations with impedance boundary conditions and divergence-free right-hand sides, with explicit dependence of the bounding constants on the problem frequency, are necessary. These results have been proved in 28]. In addition to that, an essential modification in the duality argument of [41] is required. The outcome is an estimate in a norm which is slightly weaker than $L^{2}$. As already mentioned, this analysis framework applies to any Trefftz approximation space. As an example, we consider particular plane wave spaces, for which we prove explicit $p$-convergence rates.

The outline of this paper is the following. In Section 2, we introduce the model problem, together with its variational formulation, and recall theoretical results on well-posedness, stability and regularity. The family of Trefftz-DG methods we are considering is described in Section 3 . Section 4 is devoted to the a priori error analysis (well-posedness of the discrete formulation, error estimates in a mesh-skeleton norm and in a mesh independent norm). Then, in Section 5, we consider the Trefftz-DG method based on particular plane wave spaces; we prove approximation properties of these spaces and derive convergence rates for the corresponding methods.

We end this introduction with some notation used throughout this paper. If $D$ is a domain in $\mathbb{R}^{2}$ or $\mathbb{R}^{3}$, we denote by $H^{k}(D)^{d}, d=1,2,3$, the Hilbert space with integer or fractional regularity index $k$ and valued in $\mathbb{C}^{d}$, and by $\|\cdot\|_{k, \Omega}$ the corresponding Sobolev norm; we use $H^{k}(D)=H^{k}(D)^{1}$, denote by $H_{0}^{1}(D)$ the closure in $H^{1}(D)$ of $C_{0}^{\infty}(D)$ and set $L^{2}(D)=H^{0}(D)$. 
For $D \subset \mathbb{R}^{3}$, with exterior unit normal vector to $\partial D$ denoted by $\boldsymbol{n}$, we also introduce the following spaces:

$$
\begin{aligned}
& H^{r}(\operatorname{curl} ; D)=\left\{\boldsymbol{v} \in H^{r}(D)^{3}: \nabla \times \boldsymbol{v} \in H^{r}(D)^{3}\right\}, \\
& H(\operatorname{curl} ; D)=H^{0}(\operatorname{curl} ; D), \\
& H_{0}(\operatorname{curl} ; D)=\{\boldsymbol{v} \in H(\operatorname{curl} ; D): \boldsymbol{n} \times \boldsymbol{v}=\mathbf{0} \text { on } \partial D\}, \\
& H(\operatorname{div} ; D)=\left\{\boldsymbol{v} \in L^{2}(D)^{3}: \nabla \cdot \boldsymbol{v} \in L^{2}(D)\right\}, \\
& H\left(\operatorname{div}^{0} ; D\right)=\left\{\boldsymbol{v} \in L^{2}(D)^{3}: \nabla \cdot \boldsymbol{v}=0 \text { in } D\right\}, \\
& L_{T}^{2}(\partial \Omega)=\left\{\boldsymbol{v} \in L^{2}(\partial \Omega)^{3}: \boldsymbol{v} \cdot \boldsymbol{n}=0\right\} .
\end{aligned}
$$

If $D$ is a Lipschitz domain in $\mathbb{R}^{3}$, the following integration by parts formula holds true for functions $\boldsymbol{F}, \boldsymbol{G} \in H(\operatorname{curl} ; D)$ :

$$
\int_{D} \nabla \times \boldsymbol{F} \cdot \overline{\boldsymbol{G}} \mathrm{d} V=\int_{D} \boldsymbol{F} \cdot \overline{\nabla \times \boldsymbol{G}} \mathrm{d} V+\int_{\partial D} \boldsymbol{n} \times \boldsymbol{F} \cdot \overline{\boldsymbol{G}} \mathrm{d} S,
$$

provided that the second integral on the right-hand side is read as a duality product between the appropriate trace spaces (see [13). If $\boldsymbol{F}$ is a vector-valued function defined in $D$, we denote its normal and tangential components on $\partial D$ by $\boldsymbol{F}_{N}:=$ $(\boldsymbol{F} \cdot \boldsymbol{n}) \boldsymbol{n}$ and $\boldsymbol{F}_{T}:=(\boldsymbol{n} \times \boldsymbol{F}) \times \boldsymbol{n}$, respectively. Finally, we write $B_{\gamma}\left(\boldsymbol{x}_{0}\right)$ for the (open) ball of radius $\gamma$ and centered at $\boldsymbol{x}_{0}$.

\section{The Maxwell Boundary Value problem}

Let $\Omega \subset \mathbb{R}^{3}$ be a bounded, polyhedral domain, such that

there exist a point $\boldsymbol{x}_{0} \in \Omega$ and a real number $\gamma>0$ for which $\Omega$ is star-shaped with respect to all points in $B_{\gamma}\left(\boldsymbol{x}_{0}\right)$.

Notice that this implies that $\Omega$ satisfies the uniform cone condition and thus, by 25 , Theorem 1.2.2.2], $\Omega$ is Lipschitz. We denote by $\boldsymbol{n}$ the unit normal vector field on $\partial \Omega$ pointing outside $\Omega$.

We consider the following formulation of the (homogeneous) time-harmonic Maxwell equations in terms of electric field $\boldsymbol{E}$ and magnetic field $\boldsymbol{H}$ with impedance boundary conditions in the domain $\Omega$ :

$$
\begin{cases}-i \omega \varepsilon \boldsymbol{E}-\nabla \times \boldsymbol{H}=\mathbf{0} & \text { in } \Omega, \\ -i \omega \mu \boldsymbol{H}+\nabla \times \boldsymbol{E}=\mathbf{0} & \text { in } \Omega, \\ \boldsymbol{H} \times \boldsymbol{n}-\vartheta(\boldsymbol{n} \times \boldsymbol{E}) \times \boldsymbol{n}=\boldsymbol{g} / i \omega & \text { on } \partial \Omega,\end{cases}
$$

where $\omega>0$ is a fixed wave number, and $\boldsymbol{g} \in L_{T}^{2}(\partial \Omega)$. The material coefficients $\varepsilon, \mu, \vartheta \in \mathbb{R}, \varepsilon, \mu>0$ and $\vartheta \neq 0$, are assumed to be constant in the whole domain.

By the second equation of (2.1), we can write $\boldsymbol{H}$ in terms of $\boldsymbol{E}$ as $\boldsymbol{H}=$ $(i \omega)^{-1} \mu^{-1} \nabla \times \boldsymbol{E}$; replacing this expression into the first equation and into the boundary condition, we obtain

$$
\begin{cases}\nabla \times\left(\mu^{-1} \nabla \times \boldsymbol{E}\right)-\omega^{2} \varepsilon \boldsymbol{E}=\mathbf{0} & \text { in } \Omega, \\ \left(\mu^{-1} \nabla \times \boldsymbol{E}\right) \times \boldsymbol{n}-i \omega \vartheta(\boldsymbol{n} \times \boldsymbol{E}) \times \boldsymbol{n}=\boldsymbol{g} & \text { on } \partial \Omega .\end{cases}
$$

Introducing the following subspace of $H(\operatorname{curl} ; \Omega)$ :

$$
H_{\mathrm{imp}}(\operatorname{curl} ; \Omega)=\left\{\boldsymbol{v} \in H(\operatorname{curl} ; \Omega): \boldsymbol{v}_{T} \in L_{T}^{2}(\partial \Omega)\right\},
$$


endowed with the graph norm, the variational formulation of problem (2.2) reads as follows: find $\boldsymbol{E} \in H_{\mathrm{imp}}(\operatorname{curl} ; \Omega)$ such that, for all $\boldsymbol{\xi} \in H_{\mathrm{imp}}(\operatorname{curl} ; \Omega)$, it holds that

$$
\mathcal{A}(\boldsymbol{E}, \boldsymbol{\xi})=\int_{\partial \Omega} \boldsymbol{g} \cdot \overline{\boldsymbol{\xi}}_{T} \mathrm{~d} S
$$

where

$$
\mathcal{A}(\boldsymbol{E}, \boldsymbol{\xi}):=\int_{\Omega}\left[\left(\mu^{-1} \nabla \times \boldsymbol{E}\right) \cdot(\overline{\nabla \times \boldsymbol{\xi}})-\omega^{2}(\varepsilon \boldsymbol{E}) \cdot \overline{\boldsymbol{\xi}}\right] \mathrm{d} V-i \omega \int_{\partial \Omega} \vartheta \boldsymbol{E}_{T} \cdot \overline{\boldsymbol{\xi}}_{T} \mathrm{~d} S .
$$

Under the assumptions made on $\Omega, \boldsymbol{g}$ and on the material coefficients, there exists a unique $\boldsymbol{E} \in H_{\mathrm{imp}}(\operatorname{curl} ; \Omega)$ with $\nabla \cdot(\varepsilon \boldsymbol{E})=0$ the solution to (2.3) (see [40, Thm. 4.17]). Moreover, there exists a positive constant $C_{\text {stab }}$ independent of $\omega$ but depending on $d:=\operatorname{diam}(\Omega), \gamma, \vartheta, \varepsilon$ and $\mu$, such that, if $\boldsymbol{E}$ is the solution to (2.3),

$$
\left\|\mu^{-1 / 2} \nabla \times \boldsymbol{E}\right\|_{0, \Omega}+\omega\left\|\varepsilon^{1 / 2} \boldsymbol{E}\right\|_{0, \Omega} \leq C_{\text {stab }}\|\boldsymbol{g}\|_{0, \partial \Omega}
$$

(see [28, Thm. 3.2]).

In order to develop the duality argument needed for the error analysis of Section 4.3 below, we will make use of an elliptic regularity result for the adjoint Maxwell problem:

$$
\begin{cases}\nabla \times\left(\mu^{-1} \nabla \times \boldsymbol{\Phi}\right)-\omega^{2} \varepsilon \mathbf{\Phi}=\boldsymbol{w}_{0} & \text { in } \Omega, \\ \left(\mu^{-1} \nabla \times \boldsymbol{\Phi}\right) \times \boldsymbol{n}+i \omega \vartheta(\boldsymbol{n} \times \mathbf{\Phi}) \times \boldsymbol{n}=\mathbf{0} & \text { on } \partial \Omega,\end{cases}
$$

with $\boldsymbol{w}_{0} \in H\left(\operatorname{div}^{0} ; \Omega\right)$. The variational formulation of problem (2.4) is: find $\boldsymbol{\Phi} \in$ $H_{\mathrm{imp}}(\operatorname{curl} ; \Omega)$ such that, for all $\boldsymbol{\xi} \in H_{\mathrm{imp}}(\operatorname{curl} ; \Omega)$, it holds that

$$
\begin{array}{r}
\int_{\Omega}\left[\left(\mu^{-1} \nabla \times \boldsymbol{\Phi}\right) \cdot(\overline{\nabla \times \boldsymbol{\xi}})-\omega^{2}(\varepsilon \mathbf{\Phi}) \cdot \overline{\boldsymbol{\xi}}\right] \mathrm{d} V+i \omega \int_{\partial \Omega} \vartheta \boldsymbol{\Phi}_{T} \cdot \overline{\boldsymbol{\xi}}_{T} \mathrm{~d} S \\
=\int_{\Omega} \boldsymbol{w}_{0} \cdot \overline{\boldsymbol{\xi}} \mathrm{d} V .
\end{array}
$$

Theorem 2.1 ([28, Thm. 3.2, Thm. 4.4 and Rem. 4.5]). Under the previous assumptions on $\Omega$ and on the material coefficients, for all $\boldsymbol{w}_{0} \in H\left(\operatorname{div}^{0} ; \Omega\right)$, the solution $\boldsymbol{\Phi}$ to problem (2.5) satisfies

$$
\boldsymbol{\Phi} \in H^{1 / 2+s}(\operatorname{curl} ; \Omega)
$$

for all the real parameters $s>0$ such that $s \leq \widetilde{s}$, where $0<\widetilde{s}<1 / 2$ is a parameter only depending on $\Omega$.

Moreover, there are positive constants $C_{1}$ and $C_{2}$ independent of $\omega$, but depending on $s, \Omega, \vartheta$, $\varepsilon$ and $\mu$, such that

$$
\begin{aligned}
& \|\nabla \times \boldsymbol{\Phi}\|_{0, \Omega}+\omega\|\boldsymbol{\Phi}\|_{0, \Omega} \leq C_{1}\left\|\boldsymbol{w}_{0}\right\|_{0, \Omega}, \\
& \|\nabla \times \boldsymbol{\Phi}\|_{1 / 2+s, \Omega}+\omega\|\boldsymbol{\Phi}\|_{1 / 2+s, \Omega} \leq C_{2}(1+\omega)\left\|\boldsymbol{w}_{0}\right\|_{0, \Omega} .
\end{aligned}
$$

If $\Omega$ is convex, this holds true for all $0<s<1 / 2$.

Remark 2.2. To be more precise, let $s_{\Omega}$ be the Dirichlet/Laplace regularity parameter defined in [5, Prop. 3.7] $\left(0<s_{\Omega}<1 / 2\right)$, and let $s^{*}$ be the Laplace-Beltrami regularity parameter defined in [14, Thm. 8] $\left(0<s^{*} \leq 1\right)$; then, Theorem 2.1] holds true for all real parameters $s$ such that

$$
s \leq s_{\Omega} \text { and } s<s^{*} .
$$




\section{The Trefftz-DG method}

Let $\mathcal{T}_{h}$ be a finite element partition of $\Omega$, with possible hanging nodes, of mesh width $h$ (i.e., $h=\max _{K \in \mathcal{T}_{h}} h_{K}$, with $\left.h_{K}:=\operatorname{diam}(K)\right)$ on which we will define our Trefftz-DG method; we will denote by $\mathcal{F}_{h}=\bigcup_{K \in \mathcal{T}_{h}} \partial K$ the skeleton of the mesh, and set $\mathcal{F}_{h}^{B}=\mathcal{F}_{h} \cap \partial \Omega$ and $\mathcal{F}_{h}^{I}=\mathcal{F}_{h} \backslash \mathcal{F}_{h}^{B}$.

We recall some standard DG notation. Write $\boldsymbol{n}^{+}, \boldsymbol{n}^{-}$for the exterior unit normals on $\partial K^{+}$and $\partial K^{-}$, respectively. Let $u$ and $\boldsymbol{\sigma}$ be a piecewise smooth function and vector field on $\mathcal{T}_{h}$, respectively. On $\partial K^{-} \cap \partial K^{+}$, we define

the averages: $\left\{\{u\}:=\frac{1}{2}\left(u^{+}+u^{-}\right), \quad\left\{\{\boldsymbol{\sigma}\}:=\frac{1}{2}\left(\boldsymbol{\sigma}^{+}+\boldsymbol{\sigma}^{-}\right)\right.\right.$,

the jumps: $\llbracket \boldsymbol{\sigma} \rrbracket_{T}:=\boldsymbol{n}^{+} \times \boldsymbol{\sigma}^{+}+\boldsymbol{n}^{-} \times \boldsymbol{\sigma}^{-}$.

Finally, we recall the "DG magic formula"

$$
\begin{aligned}
\sum_{K \in \mathcal{T}_{h}} \int_{\partial K} \boldsymbol{n}_{K} \times \boldsymbol{F} \cdot \overline{\boldsymbol{G}} \mathrm{d} S= & \int_{\mathcal{F}_{h}^{I}}\left(\llbracket \boldsymbol{F} \rrbracket_{T} \cdot\left\{\{\overline{\boldsymbol{G}}\}-\{\{\boldsymbol{F}\}] \cdot \llbracket \overline{\boldsymbol{G}} \rrbracket_{T}\right) \mathrm{d} S\right. \\
& +\int_{\mathcal{F}_{h}^{B}} \boldsymbol{n} \times \boldsymbol{F} \cdot \overline{\boldsymbol{G}} \mathrm{d} S
\end{aligned}
$$

thus, if $\widehat{\boldsymbol{F}}$ is a single-valued function on each edge of $\mathcal{T}_{h}$, we have

$$
\sum_{K \in \mathcal{T}_{h}} \int_{\partial K} \boldsymbol{n}_{K} \times \widehat{\boldsymbol{F}} \cdot \overline{\boldsymbol{G}} \mathrm{d} S=-\int_{\mathcal{F}_{h}^{I}} \widehat{\boldsymbol{F}} \cdot \llbracket \overline{\boldsymbol{G}} \rrbracket_{T} \mathrm{~d} S+\int_{\mathcal{F}_{h}^{B}} \boldsymbol{n} \times \widehat{\boldsymbol{F}} \cdot \overline{\boldsymbol{G}} \mathrm{d} S .
$$

We proceed by deriving our Trefftz-DG method. Set

$$
\boldsymbol{V}(K)=\left\{\boldsymbol{v} \in H(\operatorname{curl}, K), \boldsymbol{n} \times \boldsymbol{v} \in L_{T}^{2}(\partial K)\right\}
$$

Integrating by parts equation (2.1), for every $K \in \mathcal{T}_{h}$ we look for $(\boldsymbol{E}, \boldsymbol{H}) \in \boldsymbol{V}(K) \times$ $\boldsymbol{V}(K)$ such that

$$
\begin{aligned}
& i \omega \int_{K} \varepsilon \boldsymbol{E} \cdot \overline{\boldsymbol{\xi}} \mathrm{d} V+\int_{K} \boldsymbol{H} \cdot \overline{\nabla \times \boldsymbol{\xi}} \mathrm{d} V+\int_{\partial K} \boldsymbol{n} \times \boldsymbol{H} \cdot \overline{\boldsymbol{\xi}} \mathrm{d} S=0 \\
& i \omega \int_{K} \boldsymbol{H} \cdot \overline{\boldsymbol{\psi}} \mathrm{d} V-\int_{K} \boldsymbol{E} \cdot \overline{\nabla \times\left(\mu^{-1} \boldsymbol{\psi}\right)} \mathrm{d} V-\int_{\partial K} \boldsymbol{n} \times \boldsymbol{E} \cdot\left(\overline{\mu^{-1} \boldsymbol{\psi}}\right) \mathrm{d} S=0
\end{aligned}
$$

for every $(\boldsymbol{\xi}, \boldsymbol{\psi}) \in \boldsymbol{V}(K) \times \boldsymbol{V}(K)$.

Now we discretize the problem: for every $K \in \mathcal{T}_{h}$ we look for $\left(\boldsymbol{E}_{h, p}, \boldsymbol{H}_{h, p}\right) \in$ $\boldsymbol{V}_{p}^{E}(K) \times \boldsymbol{V}_{p}^{H}(K)$ such that

$$
\begin{gathered}
i \omega \int_{K} \varepsilon \boldsymbol{E}_{h, p} \cdot \overline{\boldsymbol{\xi}}_{h, p} \mathrm{~d} V+\int_{K} \boldsymbol{H}_{h, p} \cdot \overline{\nabla \times \boldsymbol{\xi}_{h, p}} \mathrm{~d} V+\int_{\partial K} \boldsymbol{n} \times \widehat{\boldsymbol{H}}_{h, p} \cdot \overline{\boldsymbol{\xi}}_{h, p} \mathrm{~d} S=0 \\
i \omega \int_{K} \boldsymbol{H}_{h, p} \cdot \overline{\boldsymbol{\psi}}_{h, p} \mathrm{~d} V-\int_{K} \boldsymbol{E}_{h, p} \cdot \overline{\nabla \times\left(\mu^{-1} \boldsymbol{\psi}_{h, p}\right)} \mathrm{d} V \\
\quad-\int_{\partial K} \boldsymbol{n} \times \widehat{\boldsymbol{E}}_{h, p} \cdot\left(\overline{\mu^{-1} \boldsymbol{\psi}_{h, p}}\right) \mathrm{d} S=0
\end{gathered}
$$

for every $\left(\boldsymbol{\xi}_{h, p}, \boldsymbol{\psi}_{h, p}\right) \in \boldsymbol{V}_{p}^{E}(K) \times \boldsymbol{V}_{p}^{H}(K)$, where $\boldsymbol{V}_{p}^{E}(K), \boldsymbol{V}_{p}^{H}(K) \subset \boldsymbol{V}(K)$ are finite dimensional spaces, and $\widehat{\boldsymbol{H}}_{h, p}$ and $\widehat{\boldsymbol{E}}_{h, p}$ on $\mathcal{F}_{h}$ are the numerical fluxes to be defined. The particular case of Trefftz-DG method which makes use of plane wave basis functions (see [34]) will be discussed in Section 5 below. 
Assuming that $\nabla \times \boldsymbol{V}_{p}^{E}(K) \subseteq \boldsymbol{V}_{p}^{H}(K)$, we can choose $\boldsymbol{\psi}_{h, p}=\nabla \times \boldsymbol{\xi}_{h, p}$ in the second equation of (3.2) and obtain

$$
\begin{aligned}
& i \omega \int_{K} \boldsymbol{H}_{h, p} \cdot \overline{\nabla \times \boldsymbol{\xi}_{h, p}} \mathrm{~d} V \\
& \quad=\int_{K} \boldsymbol{E}_{h, p} \cdot \overline{\nabla \times\left(\mu^{-1} \nabla \times \boldsymbol{\xi}_{h, p}\right)} \mathrm{d} V+\int_{\partial K} \boldsymbol{n} \times \widehat{\boldsymbol{E}}_{h, p} \cdot\left(\overline{\mu^{-1} \nabla \times \boldsymbol{\xi}_{h, p}}\right) \mathrm{d} S .
\end{aligned}
$$

Substituting this expression for $\int_{K} \boldsymbol{H}_{h, p} \cdot \overline{\nabla \times \boldsymbol{\xi}_{h, p}} \mathrm{~d} V$ into the first equation of (3.2) and multiplying by $i \omega$ give a problem in the $\boldsymbol{E}_{h, p}$ variable only: find $\boldsymbol{E}_{h, p} \in \boldsymbol{V}_{p}^{E}(K)$ such that

$$
\begin{aligned}
\int_{K} \boldsymbol{E}_{h, p} & \cdot\left(\overline{\nabla \times\left(\mu^{-1} \nabla \times \boldsymbol{\xi}_{h, p}\right)-\omega^{2} \varepsilon \boldsymbol{\xi}_{h, p}}\right) \mathrm{d} V \\
& +\int_{\partial K} \boldsymbol{n} \times \widehat{\boldsymbol{E}}_{h, p} \cdot\left(\overline{\mu^{-1} \nabla \times \boldsymbol{\xi}_{h, p}}\right) \mathrm{d} S+i \omega \int_{\partial K} \boldsymbol{n} \times \widehat{\boldsymbol{H}}_{h, p} \cdot \overline{\boldsymbol{\xi}}_{h, p} \mathrm{~d} S=0
\end{aligned}
$$

for every $\boldsymbol{\xi}_{h, p} \in \boldsymbol{V}_{p}^{E}(K)$.

The key idea of Trefftz methods is to choose $\boldsymbol{V}_{p}^{E}(K)$, which satisfies the Trefftz property

$$
\nabla \times\left(\mu^{-1} \nabla \times \boldsymbol{\xi}_{h, p}\right)-\omega^{2} \varepsilon \boldsymbol{\xi}_{h, p}=\mathbf{0} \quad \forall \boldsymbol{\xi}_{h, p} \in \boldsymbol{V}_{p}^{E}(K) .
$$

Using the Trefftz property of the test functions, the elemental equation defining the Trefftz-DG method is

$$
\int_{\partial K} \boldsymbol{n} \times \widehat{\boldsymbol{E}}_{h, p} \cdot\left(\overline{\mu^{-1} \nabla \times \boldsymbol{\xi}_{h, p}}\right) \mathrm{d} S+i \omega \int_{\partial K} \boldsymbol{n} \times \widehat{\boldsymbol{H}}_{h, p} \cdot \overline{\boldsymbol{\xi}}_{h, p} \mathrm{~d} S=0,
$$

with numerical fluxes to be defined.

Motivated by the classical UWVF 18 , and in analogy to the Helmholtz case (see [15, 29]), we define the numerical fluxes as functions on $\mathcal{F}_{h}^{I}$ :

$$
\begin{aligned}
\widehat{\boldsymbol{E}}_{h, p} & =\left\{\left\{\boldsymbol{E}_{h, p}\right\}-\frac{\beta}{i \omega} \llbracket \mu^{-1} \nabla_{h} \times \boldsymbol{E}_{h, p} \rrbracket_{T},\right. \\
\widehat{\boldsymbol{H}}_{h, p} & =\frac{1}{i \omega}\left\{\left[\mu^{-1} \nabla_{h} \times \boldsymbol{E}_{h, p}\right\}+\alpha \llbracket \boldsymbol{E}_{h, p} \rrbracket_{T},\right.
\end{aligned}
$$

and on $\mathcal{F}_{h}^{B}$ :

$$
\begin{aligned}
\widehat{\boldsymbol{E}}_{h, p} & =\boldsymbol{E}_{h, p}-\delta \vartheta^{-1}\left(\frac{1}{i \omega} \boldsymbol{n} \times\left(\mu^{-1} \nabla_{h} \times \boldsymbol{E}_{h, p}\right)+\vartheta\left(\boldsymbol{n} \times \boldsymbol{E}_{h, p}\right) \times \boldsymbol{n}+\frac{1}{i \omega} \boldsymbol{g}\right), \\
\widehat{\boldsymbol{H}}_{h, p} & =\frac{1}{i \omega \mu} \nabla_{h} \times \boldsymbol{E}_{h, p}-(1-\delta)\left(\frac{1}{i \omega \mu} \nabla_{h} \times \boldsymbol{E}_{h, p}-\vartheta\left(\boldsymbol{n} \times \boldsymbol{E}_{h, p}\right)-\frac{1}{i \omega} \boldsymbol{n} \times \boldsymbol{g}\right),
\end{aligned}
$$

where $\nabla_{h} \times \cdot$ denotes the elementwise application of the $\nabla \times \cdot$ operator, $\alpha, \beta, \delta$ are real, strictly positive, bounded functions, bounded away from zero, independent of $h, p$ and $\omega$, with $0<\delta \leq 1 / 2$.

Remark 3.1. This choice of fluxes with the parameters $\alpha, \beta$ and $\delta$ independent of the mesh size, in analogy to [15, 29, is due to the fact that our focus is on the $p$-version of the method. With a mesh-dependent choice of the flux parameters like the one made in [24] for the Helmholtz problem, one could use the same analysis technique as in [24] and possibly derive better $h$-version estimates also in the Maxwell case (see also Remark 4.11 below). 
Other numerical fluxes could also be defined by adapting to the time-harmonic Maxwell problem the DG-elliptic fluxes listed in [6] (for an example of "mixed fluxes" in the case of the Helmholtz problem, see [30]).

The above defined fluxes are single-valued on the mesh skeleton; moreover, they are consistent, i.e., replacing $\boldsymbol{E}_{h, p}$ and $\boldsymbol{H}_{h, p}$ by $\boldsymbol{E}$ and $\boldsymbol{H}$, the analytical solutions to (2.1), respectively, we have that $\widehat{\boldsymbol{E}}$ coincides with $\boldsymbol{E}$ and $\widehat{\boldsymbol{H}}$ coincides with $\boldsymbol{H}$.

Defining

$$
\boldsymbol{V}_{p}\left(\mathcal{T}_{h}\right)=\left\{\boldsymbol{\xi}_{h, p} \in L^{2}(\Omega)^{3}:\left.\boldsymbol{\xi}_{h, p}\right|_{K} \in \boldsymbol{V}_{p}^{E}(K) \forall K \in \mathcal{T}_{h}\right\},
$$

inserting the numerical fluxes into (3.3) and adding over all elements complete the definition of the Trefftz-DG method: find $\boldsymbol{E}_{h, p} \in \boldsymbol{V}_{p}\left(\mathcal{T}_{h}\right)$ such that, for all $\boldsymbol{\xi}_{h, p} \in \boldsymbol{V}_{p}\left(\mathcal{T}_{h}\right)$

$$
\mathcal{A}_{h}\left(\boldsymbol{E}_{h, p}, \boldsymbol{\xi}_{h, p}\right)=\ell_{h}\left(\boldsymbol{\xi}_{h, p}\right)
$$

where

$$
\begin{aligned}
& \mathcal{A}_{h}(\boldsymbol{E}, \boldsymbol{\xi})=-\int_{\mathcal{F}_{h}^{I}}\left\{\{\boldsymbol{E}\} \cdot \llbracket \overline{\mu^{-1} \nabla_{h} \times \boldsymbol{\xi}} \rrbracket_{T} \mathrm{~d} S-\int_{\mathcal{F}_{h}^{I}}\left\{\mu^{-1} \nabla_{h} \times \boldsymbol{E}\right\} \cdot \llbracket \overline{\boldsymbol{\xi}} \rrbracket_{T} \mathrm{~d} S\right. \\
& \quad+\int_{\mathcal{F}_{h}^{B}}(\boldsymbol{n} \times \boldsymbol{E}) \cdot\left(\overline{\mu^{-1} \nabla_{h} \times \boldsymbol{\xi}}\right) \mathrm{d} S \\
& \quad-\int_{\mathcal{F}_{h}^{B}} \delta(\boldsymbol{n} \times \boldsymbol{E}) \cdot\left(\overline{\mu^{-1} \nabla_{h} \times \boldsymbol{\xi}}\right) \mathrm{d} S-\int_{\mathcal{F}_{h}^{B}} \delta\left(\mu^{-1} \nabla_{h} \times \boldsymbol{E}\right) \cdot(\overline{\boldsymbol{n} \times \boldsymbol{\xi}}) \mathrm{d} S \\
& \quad-i \omega^{-1} \int_{\mathcal{F}_{h}^{I}} \beta \llbracket \mu^{-1} \nabla_{h} \times \boldsymbol{E} \rrbracket_{T} \cdot \llbracket \overline{\mu^{-1} \nabla_{h} \times \boldsymbol{\xi}} \rrbracket_{T} \mathrm{~d} S-i \omega \int_{\mathcal{F}_{h}^{I}} \alpha \llbracket \boldsymbol{E} \rrbracket_{T} \cdot \llbracket \overline{\boldsymbol{\xi}} \rrbracket_{T} \mathrm{~d} S \\
& \quad-i \omega^{-1} \int_{\mathcal{F}_{h}^{B}} \delta \vartheta^{-1}\left[\boldsymbol{n} \times\left(\mu^{-1} \nabla_{h} \times \boldsymbol{E}\right)\right] \cdot\left[\overline{\boldsymbol{n} \times\left(\mu^{-1} \nabla_{h} \times \boldsymbol{\xi}\right)}\right] \mathrm{d} S \\
& \quad-i \omega \int_{\mathcal{F}_{h}^{B}}(1-\delta) \vartheta(\boldsymbol{n} \times \boldsymbol{E}) \cdot(\overline{\boldsymbol{n} \times \boldsymbol{\xi}}) \mathrm{d} S
\end{aligned}
$$

and

$\ell_{h}(\boldsymbol{\xi})=\frac{1}{i \omega} \int_{\mathcal{F}_{h}^{B}} \delta \vartheta^{-1}(\boldsymbol{n} \times \boldsymbol{g}) \cdot\left(\overline{\mu^{-1} \nabla_{h} \times \boldsymbol{\xi}}\right) \mathrm{d} S+\int_{\mathcal{F}_{h}^{B}}(1-\delta)(\boldsymbol{n} \times \boldsymbol{g}) \cdot(\overline{\boldsymbol{n} \times \boldsymbol{\xi}}) \mathrm{d} S$.

The consistency of the Trefftz-DG method is a consequence of the consistency of the numerical fluxes, thus, if $\boldsymbol{E}$ is the analytical solution of (2.2), then

$$
\mathcal{A}_{h}\left(\boldsymbol{E}, \boldsymbol{\xi}_{h, p}\right)=\ell_{h}\left(\boldsymbol{\xi}_{h, p}\right) \quad \forall \boldsymbol{\xi}_{h, p} \in \boldsymbol{V}_{p}\left(\mathcal{T}_{h}\right) .
$$

Remark 3.2. The formulation of the Trefftz-DG method introduced in this section would remain unchanged if the material coefficients were piecewise constant on $\mathcal{T}_{h}$. The assumption on these coefficients to be constant in the whole domain is only required in our error analysis.

\section{THEORETICAL ANALYSIS}

In this section, we closely follow the analysis developed in [29] for the Helmholtz problem. Well-posedness and error estimates in a mesh-skeleton norm are derived exactly as in 29] (see Sections 4.1 and 4.2 below). For the derivation of error 
estimates in a mesh-independent norm, we modify the duality argument developed in [41] and used in 29] (see Section 4.3 below).

Define the broken Sobolev space

$$
H^{r}\left(\operatorname{curl} ; \mathcal{T}_{h}\right)=\left\{\boldsymbol{w} \in L^{2}(\Omega)^{3}: \boldsymbol{w}_{\left.\right|_{K}} \in H^{r}(\operatorname{curl} ; K) \quad \forall K \in \mathcal{T}_{h}\right\} .
$$

Let $\boldsymbol{T}\left(\mathcal{T}_{h}\right)$ be the piecewise Trefftz space defined on $\mathcal{T}_{h}$ by

$$
\begin{aligned}
\boldsymbol{T}\left(\mathcal{T}_{h}\right)=\{ & \left\{\boldsymbol{w} \in L^{2}(\Omega)^{3}: \exists s>0 \text { s.t. } \boldsymbol{w} \in H^{1 / 2+s}\left(\operatorname{curl} ; \mathcal{T}_{h}\right),\right. \\
& \text { and } \left.\nabla \times\left(\mu^{-1} \nabla \times \boldsymbol{w}\right)-\omega^{2} \varepsilon \boldsymbol{w}=0 \text { in each } K \in \mathcal{T}_{h}\right\} .
\end{aligned}
$$

Notice that, since $\boldsymbol{T}\left(\mathcal{T}_{h}\right) \subset H^{1 / 2+s}\left(\operatorname{curl} ; \mathcal{T}_{h}\right), s>0$, if $\boldsymbol{w} \in \boldsymbol{T}\left(\mathcal{T}_{h}\right)$, then both $\boldsymbol{n} \times \boldsymbol{w}$ and $\boldsymbol{n} \times\left(\nabla_{h} \times \boldsymbol{w}\right)$ belong to $L^{2}\left(\mathcal{F}_{h}\right)^{2}$.

We endow $\boldsymbol{T}\left(\mathcal{T}_{h}\right)$ with the mesh-skeleton norm

$$
\begin{aligned}
\left.\|\boldsymbol{w}\|\right|_{\mathcal{F}_{h}} ^{2}= & \omega^{-1}\left\|\beta^{1 / 2} \llbracket \mu^{-1} \nabla_{h} \times \boldsymbol{w} \rrbracket_{T}\right\|_{0, \mathcal{F}_{h}^{I}}^{2}+\omega\left\|\alpha^{1 / 2} \llbracket \boldsymbol{w} \rrbracket_{T}\right\|_{0, \mathcal{F}_{h}^{I}}^{2} \\
& +\omega^{-1}\left\|\delta^{1 / 2} \vartheta^{-1 / 2} \boldsymbol{n} \times\left(\mu^{-1} \nabla_{h} \times \boldsymbol{w}\right)\right\|_{0, \mathcal{F}_{h}^{B}}^{2} \\
& +\omega\left\|(1-\delta)^{1 / 2} \vartheta^{1 / 2}(\boldsymbol{n} \times \boldsymbol{w})\right\|_{0, \mathcal{F}_{h}^{B}}^{2} .
\end{aligned}
$$

If $\boldsymbol{w} \in \boldsymbol{T}\left(\mathcal{T}_{h}\right)$ and $\||\boldsymbol{w}|\|_{\mathcal{F}_{h}}=0$, then it satisfies $\boldsymbol{w} \in H_{0}(\operatorname{curl} ; \Omega), \mu^{-1} \nabla \times \boldsymbol{w} \in$ $H_{0}(\operatorname{curl} ; \Omega)$, and $\nabla \times\left(\mu^{-1} \nabla \times \boldsymbol{w}\right)-\omega^{2} \varepsilon \boldsymbol{w}=\mathbf{0}$, thus $\boldsymbol{w}=\mathbf{0}$, as a consequence of well-posedness of problem (2.2). This proves that $\||\cdot|||_{\mathcal{F}_{h}}$ is actually a norm on $\boldsymbol{T}\left(\mathcal{T}_{h}\right)$.

4.1. Well-posedness. We prove existence, uniqueness and continuous dependence on the data of solutions to Trefftz-DG methods.

Proposition 4.1. There exists a unique $\boldsymbol{E}_{h, p}$ solution to (3.4); moreover, we have continuous dependence of $\boldsymbol{E}_{h, p}$ on $\boldsymbol{g}$ :

$$
\left.\left\|\left|\boldsymbol{E}_{h, p}\right|\right\|\right|_{\mathcal{F}_{h}} \leq\left\|(1-\delta)^{1 / 2} \vartheta^{-1 / 2}(\boldsymbol{n} \times \boldsymbol{g})\right\|_{0, \mathcal{F}_{h}^{B}} .
$$

Proof. We rewrite the bilinear form $\mathcal{A}_{h}(\boldsymbol{E}, \boldsymbol{\xi})$ defined in (3.5), for all $\boldsymbol{E}, \boldsymbol{\xi} \in \boldsymbol{T}\left(\mathcal{T}_{h}\right)$ as follows: by the Trefftz property of $\boldsymbol{\xi}$, using the "DG magic formula" (3.1), for all $\boldsymbol{E}, \boldsymbol{\xi} \in \boldsymbol{T}\left(\mathcal{T}_{h}\right)$, we have

$$
\begin{aligned}
0= & \sum_{K \in \mathcal{T}_{h}} \int_{K} \boldsymbol{E} \cdot\left(\nabla \times\left(\overline{\mu^{-1} \nabla \times \boldsymbol{\xi}}\right)-\omega^{2} \varepsilon \overline{\boldsymbol{\xi}}\right) \mathrm{d} V \\
= & \sum_{K \in \mathcal{T}_{h}} \int_{K}\left(\mu^{-1} \nabla \times \boldsymbol{E} \cdot \overline{\nabla \times \boldsymbol{\xi}}-\omega^{2} \varepsilon \boldsymbol{E} \cdot \overline{\boldsymbol{\xi}}\right) \mathrm{d} V \\
& \left.-\int_{\mathcal{F}_{h}^{I}} \llbracket \boldsymbol{E} \rrbracket_{T} \cdot\left\{\overline{\mu^{-1} \nabla_{h} \times \boldsymbol{\xi}}\right\} \mathrm{d} S+\int_{\mathcal{F}_{h}^{I}}\{\boldsymbol{E}\}\right\} \cdot \llbracket \overline{\mu^{-1} \nabla_{h} \times \boldsymbol{\xi}} \rrbracket_{T} \mathrm{~d} S \\
& -\int_{\mathcal{F}_{h}^{B}}(\boldsymbol{n} \times \boldsymbol{E}) \cdot\left(\overline{\mu^{-1} \nabla_{h} \times \boldsymbol{\xi}}\right) \mathrm{d} S
\end{aligned}
$$


adding this expression of 0 to $\mathcal{A}_{h}(\boldsymbol{E}, \boldsymbol{\xi})$ gives

$$
\begin{aligned}
& \mathcal{A}_{h}(\boldsymbol{E}, \boldsymbol{\xi})=\sum_{K \in \mathcal{T}_{h}} \int_{K}\left(\mu^{-1} \nabla \times \boldsymbol{E} \cdot \overline{\nabla \times \boldsymbol{\xi}}-\omega^{2} \varepsilon \boldsymbol{E} \cdot \overline{\boldsymbol{\xi}}\right) \mathrm{d} V \\
& -\int_{\mathcal{F}_{h}^{I}} \llbracket \boldsymbol{E} \rrbracket_{T} \cdot\left\{\overline{\mu^{-1} \nabla_{h} \times \boldsymbol{\xi}}\right\} \mathrm{d} S-\int_{\mathcal{F}_{h}^{I}}\left\{\left\{\mu^{-1} \nabla_{h} \times \boldsymbol{E}\right\} \cdot \llbracket \overline{\boldsymbol{\xi}} \rrbracket_{T} \mathrm{~d} S\right. \\
& -\int_{\mathcal{F}_{h}^{B}} \delta(\boldsymbol{n} \times \boldsymbol{E}) \cdot\left(\overline{\mu^{-1} \nabla_{h} \times \boldsymbol{\xi}}\right) \mathrm{d} S-\int_{\mathcal{F}_{h}^{B}} \delta\left(\mu^{-1} \nabla_{h} \times \boldsymbol{E}\right) \cdot(\overline{\boldsymbol{n} \times \boldsymbol{\xi}}) \mathrm{d} S \\
& -i \omega^{-1} \int_{\mathcal{F}_{h}^{I}} \beta \llbracket \mu^{-1} \nabla_{h} \times \boldsymbol{E} \rrbracket_{T} \cdot \llbracket \overline{\mu^{-1} \nabla_{h} \times \boldsymbol{\xi} \rrbracket_{T}} \mathrm{~d} S-i \omega \int_{\mathcal{F}_{h}^{I}} \alpha \llbracket \boldsymbol{E} \rrbracket_{T} \cdot \llbracket \overline{\boldsymbol{\xi}} \rrbracket_{T} \mathrm{~d} S \\
& -i \omega^{-1} \int_{\mathcal{F}_{h}^{B}} \delta \vartheta^{-1}\left[\boldsymbol{n} \times\left(\mu^{-1} \nabla_{h} \times \boldsymbol{E}\right)\right] \cdot\left[\overline{\boldsymbol{n} \times\left(\mu^{-1} \nabla_{h} \times \boldsymbol{\xi}\right)}\right]_{\mathrm{d}} S \\
& -i \omega \int_{\mathcal{F}_{h}^{B}}(1-\delta) \vartheta(\boldsymbol{n} \times \boldsymbol{E}) \cdot(\overline{\boldsymbol{n} \times \boldsymbol{\xi}}) \mathrm{d} S \quad \forall \boldsymbol{E}, \boldsymbol{\xi} \in \boldsymbol{T}\left(\mathcal{T}_{h}\right) .
\end{aligned}
$$

It is immediate to see that

$$
\operatorname{Im}\left[\mathcal{A}_{h}(\boldsymbol{\xi}, \boldsymbol{\xi})\right]=-\|\boldsymbol{\xi}\| \|_{\mathcal{F}_{h}}^{2} \quad \forall \boldsymbol{\xi} \in \boldsymbol{T}\left(\mathcal{T}_{h}\right) .
$$

Existence and uniqueness of solutions to (3.4) readily follow.

By using the weighted Cauchy-Schwarz inequality and bounding $\delta$ by $1-\delta$, we obtain the following continuity property for the functional $\ell_{h}(\cdot)$ :

$$
\left|\ell_{h}(\boldsymbol{\xi})\right| \leq\left\|(1-\delta)^{1 / 2} \vartheta^{-1 / 2}(\boldsymbol{n} \times \boldsymbol{g})\right\|_{0, \mathcal{F}_{h}^{B}} \mid\|\boldsymbol{\xi}\| \|_{\mathcal{F}_{h}} \quad \forall \boldsymbol{\xi} \in \boldsymbol{T}\left(\mathcal{T}_{h}\right) .
$$

Combining (4.1) and (4.2) gives the continuous dependence of $\boldsymbol{E}_{h, p}$ on $\boldsymbol{g}$.

4.2. Error estimates in mesh-skeleton norm. By proceeding as in [24, 29, in order to prove continuity of the bilinear form $\mathcal{A}_{h}(\cdot, \cdot)$, we define the following augmented norm on $\boldsymbol{T}\left(\mathcal{T}_{h}\right)$ :

$$
\begin{aligned}
\|\boldsymbol{w}\| \|_{\mathcal{F}_{h}^{+}}^{2} & =\left.\|\boldsymbol{w}\|\right|_{\mathcal{F}_{h}} ^{2}+\omega\left\|\beta^{-1 / 2}\left\{\left\{\boldsymbol{w}_{T}\right\}\right\}\right\|_{0, \mathcal{F}_{h}^{I}}^{2} \\
& +\omega^{-1}\left\|\alpha^{-1 / 2}\left\{\left\{\left(\mu^{-1} \nabla_{h} \times \boldsymbol{w}\right)_{T}\right\}\right\}\right\|_{0, \mathcal{F}_{h}^{I}}^{2}+\omega\left\|\delta^{-1 / 2} \vartheta^{1 / 2}(\boldsymbol{n} \times \boldsymbol{w})\right\|_{0, \mathcal{F}_{h}^{B}}^{2} .
\end{aligned}
$$

Proposition 4.2. We have

$$
\left|\mathcal{A}_{h}(\boldsymbol{E}, \boldsymbol{\xi})\right| \leq 2\left\|\left.|\boldsymbol{E}|\right|_{\mathcal{F}_{h}^{+}}|| \boldsymbol{\xi} \mid\right\|_{\mathcal{F}_{h}} \quad \forall \boldsymbol{E}, \boldsymbol{\xi} \in \boldsymbol{T}\left(\mathcal{T}_{h}\right) .
$$

Proof. The result can be readily obtained from the expression (3.5) by using the weighted Cauchy-Schwarz inequality and bounding $\delta$ by $1-\delta$.

It is immediate to derive the following abstract error estimate in the $\left.\||\cdot|\|\right|_{\mathcal{F}_{h}}-$ norm.

Theorem 4.3. Assume that the analytical solution $\boldsymbol{E}$ to the Maxwell problem (2.2) belongs to $\boldsymbol{T}\left(\mathcal{T}_{h}\right)$ 1] We have

$$
||\left|\boldsymbol{E}-\boldsymbol{E}_{h, p}\right|||_{\mathcal{F}_{h}} \leq 3 \inf _{\boldsymbol{\xi}_{h, p} \in \boldsymbol{V}_{p}\left(\mathcal{T}_{h}\right)}|| \boldsymbol{E}-\boldsymbol{\xi}_{h, p}||_{\mathcal{F}_{h}^{+}} .
$$

\footnotetext{
${ }^{1}$ Whenever $\left.\boldsymbol{g}\right|_{\Gamma_{j}} \in H^{s} g\left(\Gamma_{j}\right)$ with $s_{g}>0, j=1, \ldots, m$, where $\Gamma_{1}, \ldots, \Gamma_{m}$ are the flat faces of $\partial \Omega$, then $\boldsymbol{E} \in H^{1 / 2+s}(\Omega)^{3}$ and $\nabla \times \boldsymbol{E} \in H^{1 / 2+s}(\Omega)^{3}$, for some $s>0$ which depends on $s_{g}$ and $\Omega$ (see [28 Th. 4.4]).
} 
Proof. By the triangle inequality, we can write

$$
||\left|\boldsymbol{E}-\boldsymbol{E}_{h, p}\right|||_{\mathcal{F}_{h}} \leq||\left|\boldsymbol{E}-\boldsymbol{\xi}_{h, p}\right|||_{\mathcal{F}_{h}}+||\left|\boldsymbol{\xi}_{h, p}-\boldsymbol{E}_{h, p}\right|||_{\mathcal{F}_{h}} \quad \forall \boldsymbol{\xi}_{h, p} \in \boldsymbol{V}_{p}\left(\mathcal{T}_{h}\right) ;
$$

we only need to prove that ||$\left|\boldsymbol{\xi}_{h, p}-\boldsymbol{E}_{h, p}\right|\left\|_{\mathcal{F}_{h}} \leq 3\right\|\left|\boldsymbol{E}-\boldsymbol{\xi}_{h, p}\right| \|_{\mathcal{F}_{h}^{+}}$.

Since $\boldsymbol{\xi}_{h, p}-\boldsymbol{E}_{h, p} \in \boldsymbol{T}\left(\mathcal{T}_{h}\right)$, then

$$
\| \boldsymbol{\xi}_{h, p}-\boldsymbol{E}_{h, p}||_{\mathcal{F}_{h}}^{2}=-\operatorname{Im}\left[\mathcal{A}_{h}\left(\boldsymbol{\xi}_{h, p}-\boldsymbol{E}_{h, p}, \boldsymbol{\xi}_{h, p}-\boldsymbol{E}_{h, p}\right)\right],
$$

and by the Galerkin orthogonality and the continuity stated in Proposition 4.2 we obtain

$$
||\left|\boldsymbol{\xi}_{h, p}-\boldsymbol{E}_{h, p}\right|||_{\mathcal{F}_{h}}^{2} \leq\left. 2||\left|\boldsymbol{E}-\boldsymbol{\xi}_{h, p}\right|\right|_{\mathcal{F}_{h}^{+}}||\left|\boldsymbol{\xi}_{h, p}-\boldsymbol{E}_{h, p}\right|||_{\mathcal{F}_{h}}
$$

which concludes the proof.

Remark 4.4. The error bounds in Theorem 4.3 and Theorem 4.9 below are proved under minimal regularity assumptions on the analytical solutions, namely, $H^{\frac{1}{2}+s}$, $s>0$. This indicates that the considered methods are not affected by so-called spurious solutions (i.e., numerical solutions which converge to non-physical solutions; for discretizations to the Maxwell problem, this might occur in the case of singularities).

On the other hand, Theorem 4.3 guarantees $p$-convergence of Trefftz-DG methods of the type considered in this paper only provided that the spaces $\boldsymbol{V}_{p}\left(\mathcal{T}_{h}\right)$ are such that

$$
\lim _{p \rightarrow+\infty} \inf _{\boldsymbol{\xi}_{h, p} \in \boldsymbol{V}_{p}\left(\mathcal{T}_{h}\right)}\|\| \boldsymbol{E}-\boldsymbol{\xi}_{h, p} \|_{\mathcal{F}_{h}^{+}}=0
$$

Thus, possible restrictions on the solution smoothness to prove convergence of a given Trefftz-DG method are not due to the analysis framework, but would only depend on the choice of the approximation spaces.

4.3. Error estimates in a mesh-independent norm. For the Helmholtz problem, error estimates in the $L^{2}$-norm were derived in [15, 29] from error estimates in mesh skeleton norms, by bounding the $L^{2}$-norms of Trefftz functions by their mesh skeleton norms. This was carried out by using a modified duality argument developed in [41].

The first problem in repeating that argument for the time-harmonic Maxwell problem consists of the lack of stability estimates for the dual problem with a generic (non-divergence-free) $\boldsymbol{w} \in \boldsymbol{T}\left(\mathcal{T}_{h}\right)$ on the right-hand side (see [28]). In order to overcome this problem, we will consider the $L^{2}$-orthogonal Helmholtz decomposition of $\boldsymbol{w}$,

$$
\boldsymbol{w}=\boldsymbol{w}_{0}+\nabla p
$$

with $\boldsymbol{w}_{0} \in H\left(\operatorname{div}^{0} ; \Omega\right)$ and $p \in H_{0}^{1}(\Omega)$ (see, e.g., [40, Theorem 3.45]), and estimate $\boldsymbol{w}_{0}$ and $\nabla p$ separately.

An estimate of $\boldsymbol{w}_{0}$ in the $L^{2}$-norm can be obtained by proceeding as in [15, 29, while the poor regularity of $p$, and here comes the second problem, does not allow us to obtain an $L^{2}$-norm estimate of $\nabla p$ (and thus of $\boldsymbol{w}$ ).

For this reason, we introduce the following weaker norm: for every $\boldsymbol{u} \in L^{2}(\Omega)^{3}$, we define

$$
\|\boldsymbol{u}\|_{H(\operatorname{div} ; \Omega)^{\prime}}:=\sup _{\boldsymbol{v} \in H(\operatorname{div} ; \Omega)} \frac{\int_{\Omega} \boldsymbol{u} \cdot \overline{\boldsymbol{v}} \mathrm{d} V}{\|\boldsymbol{v}\|_{H(\operatorname{div} ; \Omega)}}
$$


where $\|\boldsymbol{v}\|_{H(\operatorname{div} ; \Omega)}^{2}=\|\boldsymbol{v}\|_{0, \Omega}^{2}+\operatorname{diam}(\Omega)^{2}\|\nabla \cdot \boldsymbol{v}\|_{0, \Omega}^{2}$. Notice that, for every $\boldsymbol{u} \in$ $H\left(\operatorname{div}^{0} ; \Omega\right),\|\boldsymbol{u}\|_{H(\operatorname{div} ; \Omega)^{\prime}}=\|\boldsymbol{u}\|_{0, \Omega}$.

In the following, we bound the $L^{2}$-norm of $\boldsymbol{w}_{0}$ and the $H(\operatorname{div} ; \Omega)^{\prime}$-norm of $\nabla p$ by the $\left|\|\cdot \mid\|_{\mathcal{F}_{h}}-\right.$ norm of $\boldsymbol{w}$ (see Propositions 4.5 and 4.7 below). Then, error estimates of the Trefftz-DG methods presented in this paper in the $H(\operatorname{div} ; \Omega)^{\prime}-$ norm will follow from error estimates in the $\||\cdot|\|_{\mathcal{F}_{h}}$-norm. These final estimates are reported in Theorem 4.9 below.

We define two mesh parameters which will enter the constants in the error estimates: the shape regularity measure

$$
\operatorname{s.r.}\left(\mathcal{T}_{h}\right):=\max _{K \in \mathcal{T}_{h}} \frac{h_{K}}{d_{K}},
$$

where $d_{K}$ is the diameter of the largest ball contained in $K$, and the quasi-uniformity measure

$$
q . u .\left(\mathcal{T}_{h}\right):=\max _{K \in \mathcal{T}_{h}} \frac{h}{h_{K}} .
$$

As mentioned before, we bound $\left\|\boldsymbol{w}_{0}\right\|_{0, \Omega}$ by a modified duality argument.

Proposition 4.5. Let $\boldsymbol{w} \in \boldsymbol{T}\left(\mathcal{T}_{h}\right)$ and let $\boldsymbol{w}_{0} \in H\left(\operatorname{div}^{0} ; \Omega\right)$ be its first component in decomposition (4.3). Then, there exists a positive constant $C$ independent of $\boldsymbol{w}$, $h, p$ and $\omega$ such that

$$
\left\|\boldsymbol{w}_{0}\right\|_{0, \Omega} \leq C\left[\omega^{-1 / 2} h^{-1 / 2}+\omega^{-1 / 2} h^{s}+\omega^{1 / 2} h^{s}\right]\|\boldsymbol{w}\|_{\mathcal{F}_{h}}
$$

for all real parameters $s>0$ satisfying the upper bound in Theorem 2.1. The constant $C$ depends on $\Omega, s$, s.r. $\left(\mathcal{T}_{h}\right)$, q.u. $\left(\mathcal{T}_{h}\right), \vartheta, \mu$, and on the flux parameters.

Proof. Consider the adjoint problem (2.4), and let $\boldsymbol{\Phi}$ be its solution (with source term $\boldsymbol{w}_{0}$ ). Since, due to the $L^{2}$-orthogonality of decomposition (4.3),

$$
\left\|\boldsymbol{w}_{0}\right\|_{0, \Omega}^{2}=\int_{\Omega} \boldsymbol{w}_{0} \cdot \overline{\boldsymbol{w}} \mathrm{d} V
$$

by multiplying the first equation of problem (2.4) by $\boldsymbol{w}$, integrating by parts twice and taking into account that $\boldsymbol{w}$ is a Trefftz function, we have

$$
\begin{aligned}
\int_{\Omega} \boldsymbol{w}_{0} \cdot \overline{\boldsymbol{w}} \mathrm{d} V= & \sum_{K \in \mathcal{T}_{h}} \int_{\partial K} \boldsymbol{n} \times \boldsymbol{\Phi} \cdot\left(\overline{\mu^{-1} \nabla \times \boldsymbol{w}}\right) \mathrm{d} S \\
& +\sum_{K \in \mathcal{T}_{h}} \int_{\partial K} \boldsymbol{n} \times\left(\mu^{-1} \nabla \times \mathbf{\Phi}\right) \cdot \overline{\boldsymbol{w}} \mathrm{d} S \\
= & -\int_{\mathcal{F}_{h}^{I}}\left(\boldsymbol{\Phi} \cdot \llbracket \overline{\mu^{-1} \nabla_{h} \times \boldsymbol{w}} \rrbracket_{T}+\left(\mu^{-1} \nabla \times \mathbf{\Phi}\right) \cdot \llbracket \overline{\boldsymbol{w}} \rrbracket_{T}\right) \mathrm{d} S \\
& +\int_{\mathcal{F}_{h}^{B}}\left(\boldsymbol{n} \times \mathbf{\Phi} \cdot\left(\overline{\mu^{-1} \nabla_{h} \times \boldsymbol{w}}\right)+\boldsymbol{n} \times\left(\mu^{-1} \nabla \times \mathbf{\Phi}\right) \cdot \overline{\boldsymbol{w}}\right) \mathrm{d} S .
\end{aligned}
$$

The boundary condition in the second equation of (2.4) implies that

$$
\boldsymbol{n} \times\left(\mu^{-1} \nabla \times \boldsymbol{\Phi}\right) \cdot \overline{\boldsymbol{w}}=i \omega \vartheta(\boldsymbol{n} \times \boldsymbol{\Phi}) \cdot(\overline{\boldsymbol{n} \times \boldsymbol{w}}) ;
$$


using this and the weighted Cauchy-Schwarz inequality, together with $(1-\delta)^{-1 / 2} \leq$ $\delta^{-1 / 2}$, and the definition of the $\left|\|\cdot \mid\|_{\mathcal{F}_{h}}\right.$-norm, we get

$$
\begin{aligned}
\left|\int_{\Omega} \boldsymbol{w}_{0} \cdot \overline{\boldsymbol{w}} \mathrm{d} V\right| \leq & {\left[\sum_{f \in \mathcal{F}_{h}^{I}}\left(\omega\left\|\beta^{-1 / 2} \boldsymbol{n} \times \boldsymbol{\Phi}\right\|_{0, f}^{2}+\omega^{-1}\left\|\alpha^{-1 / 2} \boldsymbol{n} \times\left(\mu^{-1} \nabla \times \boldsymbol{\Phi}\right)\right\|_{0, f}^{2}\right)\right.} \\
& \left.+\sum_{f \in \mathcal{F}_{h}^{B}} \omega\left\|\delta^{-1 / 2} \vartheta^{1 / 2} \boldsymbol{n} \times \boldsymbol{\Phi}\right\|_{0, f}^{2}\right]^{1 / 2}\|\boldsymbol{w}\| \|_{\mathcal{F}_{h}} \\
= & : \mathcal{G}(\boldsymbol{\Phi})^{1 / 2}\|\boldsymbol{w}\| \|_{\mathcal{F}_{h}} .
\end{aligned}
$$

Defining $\zeta$ on $\mathcal{F}_{h}$ by $\zeta=\beta$ if $f \in \mathcal{F}_{h}^{I}$ and $\zeta=\delta \vartheta^{-1}$ if $f \in \mathcal{F}_{h}^{B}$, we can write

$$
\mathcal{G}(\mathbf{\Phi}) \leq \sum_{K \in \mathcal{T}_{h}}\left(\omega\left\|\zeta^{-1 / 2} \boldsymbol{n} \times \boldsymbol{\Phi}\right\|_{0, \partial K}^{2}+\omega^{-1}\left\|\alpha^{-1 / 2} \boldsymbol{n} \times\left(\mu^{-1} \nabla \times \boldsymbol{\Phi}\right)\right\|_{0, \partial K}^{2}\right) .
$$

For any $K \in \mathcal{T}_{h}$, the trace inequality

$$
\|u\|_{0, \partial K}^{2} \leq C\left(h_{K}^{-1}\|u\|_{0, K}^{2}+h_{K}^{2 \eta}|u|_{1 / 2+\eta, K}^{2}\right) \quad \forall u \in H^{1 / 2+\eta}(K)
$$

holds provided that $\eta>0$, with $C>0$ only depending on the shape of $K$ and on $\eta$ (see [37, Th. A.2]). Since, from Theorem 2.1, $\mathbf{\Phi}$ belongs to $H^{1 / 2+s}(\operatorname{curl} ; \Omega$ ) for all $s>0$ satisfying the upper bound in Theorem 2.1. using the previous trace inequality and taking into account that the material coefficients are constant, we get

$$
\begin{aligned}
\mathcal{G}(\boldsymbol{\Phi}) \leq C & {\left[\omega h^{-1}\|\boldsymbol{\Phi}\|_{0, \Omega}^{2}+\omega h^{2 s}\|\boldsymbol{\Phi}\|_{1 / 2+s, \Omega}^{2}\right.} \\
& \left.+\omega^{-1} h^{-1}\|\nabla \times \boldsymbol{\Phi}\|_{0, \Omega}^{2}+\omega^{-1} h^{2 s}\|\nabla \times \mathbf{\Phi}\|_{1 / 2+s, \Omega}^{2}\right],
\end{aligned}
$$

with the constant $C>0$ independent of $h, p$ and $\omega$, but depending on $s, \mu, \operatorname{s.r} .\left(\mathcal{T}_{h}\right)$, q.u. $\left(\mathcal{T}_{h}\right)$, and on the flux parameters. Using the stability estimates in Theorem 2.1 , we obtain

which gives the result.

$$
\mathcal{G}(\boldsymbol{\Phi}) \leq C\left[\omega^{-1} h^{-1}+\omega^{-1} h^{2 s}+\omega h^{2 s}\right]\left\|\boldsymbol{w}_{0}\right\|_{0, \Omega}^{2},
$$

Before deriving an estimate for the component $\nabla p$ of decomposition (4.3), we recall the following regularity result (see [26]).

Lemma 4.6 ([26, Cor. 2.6.7, Cor. 2.6.8]). Under our assumptions on $\Omega$, there exists $\eta^{*}, 0<\eta^{*} \leq 1 / 2$, only depending on $\Omega$ such that, for all $q \in H_{0}^{1}(\Omega)$ satisfying $\Delta q \in L^{2}(\Omega)$, we have that $q$ belongs to $H^{3 / 2+\eta}(\Omega)$ for all $\eta<\eta^{*}$ and

$$
|q|_{3 / 2+\eta, \Omega} \leq C\|\Delta q\|_{0, \Omega}^{2}
$$

with a positive constant $C$ only depending on $\Omega$ and on $\eta$. If $\Omega$ is convex, this holds true for all $0<\eta \leq 1 / 2$.

Proposition 4.7. Let $\boldsymbol{w} \in \boldsymbol{T}\left(\mathcal{T}_{h}\right)$ and let $p \in H_{0}^{1}(\Omega)$ be the second component of its decomposition (4.3). Then, there exists a positive constant $C$ independent of $\boldsymbol{w}$, $h, p$ and $\omega$, but depending on $\Omega, \varepsilon$, and on the flux parameter $\beta$, such that

$$
\|\nabla p\|_{H(\operatorname{div} ; \Omega)^{\prime}} \leq C \omega^{-3 / 2}\left(h^{-1 / 2}+h^{\eta}\right)\|\| \boldsymbol{w} \|_{\mathcal{F}_{h}}
$$

for all $\eta>0$ satisfying the upper bounds in Lemma 4.6. 
Proof. Let $\boldsymbol{w} \in \boldsymbol{T}\left(\mathcal{T}_{h}\right)$ and let $q \in H_{0}^{1}(\Omega)$ be as in Lemma 4.6, i.e., $\Delta q \in L^{2}(\Omega)$; we have

$$
\begin{aligned}
& \left|\int_{\Omega} \boldsymbol{w} \cdot \nabla \bar{q} \mathrm{~d} V\right|=\left|\sum_{K \in \mathcal{T}_{h}} \int_{K} \frac{1}{\omega^{2} \varepsilon} \nabla_{h} \times\left(\mu^{-1} \nabla_{h} \times \boldsymbol{w}\right) \cdot \nabla \bar{q} \mathrm{~d} V\right| \\
& =\frac{1}{\omega^{2} \varepsilon}\left|\sum_{K \in \mathcal{T}_{h}} \int_{\partial K} \boldsymbol{n} \times\left(\mu^{-1} \nabla_{h} \times \boldsymbol{w}\right) \cdot \nabla \bar{q} \mathrm{~d} S\right| \\
& \nabla q \in H(\operatorname{curl} ; \Omega), \\
& \stackrel{q \in H_{0}^{1}(\Omega)}{=} \quad \frac{1}{\omega^{2} \varepsilon}\left|\int_{\mathcal{F}_{h}^{I}} \llbracket \mu^{-1} \nabla_{h} \times \boldsymbol{w} \rrbracket_{T} \cdot \nabla \bar{q} \mathrm{~d} S\right| \\
& \leq \frac{1}{\omega^{3 / 2} \varepsilon \beta_{\min }^{1 / 2}}|\|\boldsymbol{w}\||_{\mathcal{F}_{h}}\|\nabla q\|_{0, \mathcal{F}_{h}^{I}} \\
& \stackrel{\text { (4.4) }}{\leq} \frac{C}{\omega^{3 / 2} \varepsilon \beta_{\min }^{1 / 2}}|||\boldsymbol{w}|||_{\mathcal{F}_{h}}\left(h^{-1 / 2}|q|_{1, \Omega}+h^{\eta}|q|_{3 / 2+\eta, \Omega}\right) \\
& \leq \frac{C\left(h^{-\frac{1}{2}}+h^{\eta}\right)}{\omega^{3 / 2} \varepsilon \beta_{\min }^{1 / 2}}\|\boldsymbol{w}\|\left\|_{\mathcal{F}_{h}}\right\| \Delta q \|_{0, \Omega},
\end{aligned}
$$

with $\beta_{\min }:=\min _{\boldsymbol{x} \in \mathcal{F}_{h}^{I}} \beta$, and the positive constant $C$ only depending on $\Omega$.

Given a function $\boldsymbol{v} \in H(\operatorname{div} ; \Omega)$, consider its $L^{2}$-orthogonal Helmholtz decomposition $\boldsymbol{v}=\boldsymbol{v}_{0}+\nabla q_{\boldsymbol{v}}$ with $\boldsymbol{v}_{0} \in H\left(\operatorname{div}^{0} ; \Omega\right)$ and $q_{\boldsymbol{v}} \in H_{0}^{1}(\Omega)$; then, $\Delta q_{\boldsymbol{v}}=\nabla \cdot \boldsymbol{v}$ and $\left(\nabla q^{\prime}, \boldsymbol{v}_{0}\right)=0$ for every $q^{\prime} \in H_{0}^{1}(\Omega)$. This allows us to derive the desired bound:

$$
\begin{aligned}
& \|\nabla p\|_{H(\operatorname{div}, \Omega)^{\prime}}=\sup _{\boldsymbol{v} \in H(\operatorname{div}, \Omega)} \frac{\int_{\Omega} \nabla p \cdot \overline{\boldsymbol{v}} \mathrm{d} V}{\|\boldsymbol{v}\|_{H(\operatorname{div}, \Omega)}} \\
& \stackrel{\sqrt[4.3]{=}}{=} \sup _{\boldsymbol{v} \in H(\operatorname{div}, \Omega)} \frac{\int_{\Omega} \nabla p \cdot \overline{\boldsymbol{v}}_{0} \mathrm{~d} V+\int_{\Omega}\left(\boldsymbol{w}-\boldsymbol{w}_{0}\right) \cdot \nabla \bar{q}_{\boldsymbol{v}} \mathrm{d} V}{\|\boldsymbol{v}\|_{H(\operatorname{div}, \Omega)}} \\
& \int_{\Omega} \nabla q^{\prime} \cdot \overline{\boldsymbol{v}}_{0} \mathrm{~d} V=\left(\boldsymbol{w}_{0}, \nabla q^{\prime}\right)=0, \\
& \forall q^{\prime} \in H_{0}^{1}(\Omega) \quad \sup _{\boldsymbol{v} \in H(\operatorname{div}, \Omega)} \frac{\int_{\Omega} \boldsymbol{w} \cdot \nabla \bar{q}_{\boldsymbol{v}} \mathrm{d} V}{\|\boldsymbol{v}\|_{H(\operatorname{div}, \Omega)}} \\
& \stackrel{\sqrt{4.5}}{\leq} \frac{C\left(h^{-\frac{1}{2}}+h^{\eta}\right)}{\omega^{3 / 2} \varepsilon \beta_{\min }^{1 / 2}}\|\boldsymbol{w}\|_{\mathcal{F}_{h}} \sup _{\boldsymbol{v} \in H(\operatorname{div}, \Omega)} \frac{\left\|\Delta q_{\boldsymbol{v}}\right\|_{0, \Omega}}{\|\boldsymbol{v}\|_{H(\operatorname{div}, \Omega)}} \\
& \Delta q_{\boldsymbol{v}} \equiv \nabla \cdot \boldsymbol{v} \frac{C\left(h^{-\frac{1}{2}}+h^{\eta}\right)}{\omega^{3 / 2} \varepsilon \beta_{\min }^{1 / 2}}\|\boldsymbol{w}\| \|_{\mathcal{F}_{h}} \sup _{\boldsymbol{v} \in H(\operatorname{div}, \Omega)} \frac{\|\nabla \cdot \boldsymbol{v}\|_{0, \Omega}}{\|\boldsymbol{v}\|_{H(\operatorname{div}, \Omega)}} \\
& \leq \frac{C\left(h^{-\frac{1}{2}}+h^{\eta}\right)}{\omega^{3 / 2} \varepsilon \beta_{\min }^{1 / 2}}\||\boldsymbol{w}|\|_{\mathcal{F}_{h}} .
\end{aligned}
$$

We have the following result.

Proposition 4.8. Let $\boldsymbol{w} \in \boldsymbol{T}\left(\mathcal{T}_{h}\right)$. Under our assumptions on $\Omega$ and on the material coefficients, there exists a positive constant $C$ independent of $\boldsymbol{w}, h, p$ and $\omega$ such that

with

$$
\|\boldsymbol{w}\|_{H(\operatorname{div} ; \Omega)^{\prime}} \leq C f(\omega, h)\|\| \boldsymbol{w} \|\left.\right|_{\mathcal{F}_{h}}
$$

$$
f(\omega, h):=\left[\omega^{-1 / 2} h^{-1 / 2}+\omega^{-1 / 2} h^{s}+\omega^{1 / 2} h^{s}+\omega^{-3 / 2}\left(h^{-1 / 2}+h^{\eta}\right)\right],
$$


for all $s>0$ and $\eta>0$ satisfying the upper bounds in Theorem 2.1 and Lemma 4.6, respectively. The constant $C$ depends on $\Omega, s, \eta, s . r .\left(\mathcal{T}_{h}\right), q . u .\left(\mathcal{T}_{h}\right), \vartheta, \varepsilon, \mu$, and on the flux parameters.

Proof. By using the properties of the Helmholtz decomposition (4.3), we have

$$
\|\boldsymbol{w}\|_{H(\operatorname{div}, \Omega)^{\prime}} \leq\left\|\boldsymbol{w}_{0}\right\|_{0, \Omega}+\|\nabla p\|_{H(\operatorname{div}, \Omega)^{\prime}} .
$$

The result follows from Proposition 4.5 and Proposition 4.7

The main result of this section directly follows from Theorem 4.3 and Proposition 4.8

Theorem 4.9. In addition to our assumptions on $\Omega, \boldsymbol{g}$ and on the material coefficients, assume that the analytical solution $\boldsymbol{E}$ to the Maxwell problem (2.2) belongs to $\boldsymbol{T}\left(\mathcal{T}_{h}\right)$. Then there exists a positive constant $C$ independent of $h, p$ and $\omega$ such that

$$
\left\|\boldsymbol{E}-\boldsymbol{E}_{h, p}\right\|_{H(\operatorname{div} ; \Omega)^{\prime}} \leq C f(\omega, h) \inf _{\boldsymbol{\xi}_{h, p} \in \boldsymbol{V}_{p}\left(\mathcal{T}_{h}\right)}\left\|\boldsymbol{E}-\boldsymbol{\xi}_{h, p} \mid\right\|_{\mathcal{F}_{h}^{+}},
$$

with $f(\omega, h)$ given by (4.6), for all $s>0$ and $\eta>0$ satisfying the upper bounds in Theorem 2.1 and Lemma 4.6, respectively. The constant $C$ depends on $\Omega, s, \eta$, s.r. $\left(\mathcal{T}_{h}\right), q . u .\left(\mathcal{T}_{h}\right), \vartheta, \varepsilon, \mu$, and on the flux parameters,

Remark 4.10. In order to apply the estimate of Theorem 4.9 in the case of locally refined meshes, we have to dispense with the "quasi-uniformity assumption", that is, the dependence of the constants on q.u. $\left(\mathcal{T}_{h}\right)$. In an attempt to achieve this, one may link the coefficients $\alpha, \beta$, and $\delta$ in the definition of the numerical fluxes $\widehat{\boldsymbol{E}}_{h, p}$ and $\widehat{\boldsymbol{H}}_{h, p}$ to the local mesh size $h_{K}$, hoping to offset the negative powers of $h_{K}$ in the trace inequality (4.4). However, the constraint $\delta \leq \frac{1}{2}$ thwarts this idea.

Remark 4.11. The error estimate given in Theorem 4.9 should not be considered as an $h$-version error estimate. Indeed, as already mentioned in Remark 3.1, one could adapt to the Maxwell problem the mesh size dependent numerical fluxes and the analysis framework developed in 24] for the Helmholtz equation. In this way, one should obtain better estimates, namely, with no negative powers of $h$ in the expression of $f(\omega, h)$, provided that a threshold condition is satisfied.

\section{ThE PWDG METHOD}

We denote by Plane Wave Discontinuous Galerkin (PWDG) method the particular Trefftz-DG method which makes use of plane wave basis functions. Vectorvalued plane waves are vector field defined as $\boldsymbol{x} \mapsto \boldsymbol{a} e^{i \omega \sqrt{\varepsilon \mu} \boldsymbol{d} \cdot \boldsymbol{x}}$, where $\boldsymbol{a}$ and $\boldsymbol{d}$ are constant unit vectors. They are componentwise solutions to the Helmholtz equation and they are the solution to the Maxwell equation if and only if $\boldsymbol{a} \cdot \boldsymbol{d}=0$.

We define local plane wave approximation spaces in a slightly different way than the one in 34. Given an integer $q \geq 1$, introduce a set of $p=(q+1)^{2}$ plane wave propagation directions

$$
\left\{\boldsymbol{d}_{\ell}\right\}_{1 \leq \ell \leq p},
$$

together with the associated set of $2 p$ pairs of directions:

$$
\begin{gathered}
d_{2 p}(K)=\left\{\left(\boldsymbol{d}_{\ell}, \boldsymbol{a}_{\ell}^{i}\right)_{\substack{1 \leq \ell \leq p \\
i=1,2}} \in \mathbb{R}^{3} \times \mathbb{R}^{3},\left|\boldsymbol{d}_{\ell}\right|=\left|\boldsymbol{a}_{\ell}^{i}\right|=1,\right. \\
\left.\left(\boldsymbol{d}_{\ell}, \boldsymbol{a}_{\ell}^{1}\right)_{\mathbb{R}^{3}}=0, \boldsymbol{a}_{\ell}^{2}=\boldsymbol{a}_{\ell}^{1} \times \boldsymbol{d}_{\ell}\right\} .
\end{gathered}
$$


Then, we define $\boldsymbol{V}_{2 p}^{E}(K)$ as

$$
\boldsymbol{V}_{2 p}^{E}(K)=\left\{\sum_{\substack{1 \leq \ell \leq p \\ i=1,2}} \alpha_{\ell}^{i} \boldsymbol{a}_{\ell}^{i} e^{i \omega \sqrt{\varepsilon \mu} \boldsymbol{d}_{\ell} \cdot \boldsymbol{x}},\left(\boldsymbol{d}_{\ell}, \boldsymbol{a}_{\ell}^{i}\right)_{\substack{1 \leq \ell \leq p \\ i=1,2}} \in d_{2 p}(K), \alpha_{\ell}^{i} \in \mathbb{C}\right\},
$$

where $\boldsymbol{a}_{\ell}^{i}, i=1,2$, represent the polarization directions of the plane wave propagating along $\boldsymbol{d}_{\ell}$.

Finally, we define the discrete Maxwell-Trefftz spaces $\boldsymbol{V}_{2 p}\left(\mathcal{T}_{h}\right) \subset \boldsymbol{T}\left(\mathcal{T}_{h}\right)$ :

$$
\boldsymbol{V}_{2 p}\left(\mathcal{T}_{h}\right)=\left\{\boldsymbol{\xi}_{h, 2 p} \in L^{2}(\Omega)^{3}:\left.\boldsymbol{\xi}_{h, 2 p}\right|_{K} \in \boldsymbol{V}_{2 p}^{E}(K) \forall K \in \mathcal{T}_{h}\right\}
$$

In Section [5.1. we derive approximation estimates of homogeneous Maxwell solutions in the vector-valued plane wave approximation spaces $\boldsymbol{V}_{2 p}\left(\mathcal{T}_{h}\right)$. Then, in Section [5.2, we will insert these estimates into Theorem 4.3 and Theorem 4.9 in order to derive convergence rates of the PWDG method for problem (2.2).

5.1. Approximation properties of plane wave spaces. The strategy we use to derive approximation estimates of homogeneous Maxwell solutions $\boldsymbol{E}$ in $\boldsymbol{V}_{2 p}\left(\mathcal{T}_{h}\right)$ is to approximate $\boldsymbol{E}$ as the curl of $\boldsymbol{H}:=(i \omega)^{-1} \mu^{-1} \nabla \times \boldsymbol{E}$ (thus, $\boldsymbol{E}=-(i \omega)^{-1} \varepsilon^{-1} \nabla \times$ $\boldsymbol{H})$. We have that not only $\boldsymbol{H}$ is a Maxwell-Trefftz function, but its components are Helmholtz-Trefftz functions. Thus, we can exploit the best approximation estimates for (scalar) homogeneous Helmholtz solutions in Helmholtz-Trefftz spaces obtained in 38] in order to approximate $\boldsymbol{H}$ in a larger space than the Maxwell-Trefftz space $\boldsymbol{V}_{2 p}\left(\mathcal{T}_{h}\right)$. On the other hand, one can find a basis for this larger space formed by three vector functions: two of them generate $\boldsymbol{V}_{2 p}\left(\mathcal{T}_{h}\right)$, while the third one generates a space of non-Trefftz but curl-free functions; this allows us to find approximation estimates for the curl of $\boldsymbol{H}$, and thus for $\boldsymbol{E}$, in $\boldsymbol{V}_{2 p}\left(\mathcal{T}_{h}\right)$.

Remark 5.1. The fact that we approximate $\boldsymbol{E}$ as the curl of $\boldsymbol{H}$ leads to estimates which are one order lower than expected. In order to derive sharper best approximation estimates, one might think of extending to the Maxwell problem the approach of [38, 39], based on Vekua's theory. This will be the subject of future investigation.

Before stating our approximation result, we introduce some further notation and assumptions.

Assume that

all elements in $\mathcal{T}_{h}$ have Lipschitz boundaries, and there exist $\rho \in$ $(0,1 / 2]$ and $\rho_{0} \in(0, \rho]$ such that every $K \in \mathcal{T}_{h}$ contains the ball $B_{\rho h_{K}}\left(\boldsymbol{x}_{K}\right)$ (shape regularity), and is star-shaped with respect to the ball $B_{\rho_{0} h_{K}}\left(\boldsymbol{x}_{K}\right)$, for some $\boldsymbol{x}_{K} \in K$.

We point out that, whenever $\mathcal{T}_{h}$ is made of convex polyhedra, the previous assumption is satisfied.

We fix $q, r \in \mathbb{N}$, and set $p=(q+1)^{2}$. Let $K \in \mathcal{T}_{h}$, and let $\boldsymbol{E} \in H^{r+1}(\operatorname{curl} ; K)$ be a solution of

$$
\nabla \times\left(\mu^{-1} \nabla \times \boldsymbol{E}\right)-\omega^{2} \varepsilon \boldsymbol{E}=0 \quad \text { in } K .
$$

Define $\boldsymbol{H}:=(i \omega)^{-1} \mu^{-1} \nabla \times \boldsymbol{E}$. Then

$$
\nabla \times\left(\varepsilon^{-1} \nabla \times \boldsymbol{H}\right)-\omega^{2} \mu \boldsymbol{H}=0 \quad \text { in } K,
$$


which, since $\varepsilon$ and $\mu$ are constant, $\nabla \times \nabla \times \boldsymbol{H}=\nabla(\nabla \cdot \boldsymbol{H})-\Delta \boldsymbol{H}$ and $\nabla \cdot \boldsymbol{H}=0$, can be rewritten as

$$
\Delta \boldsymbol{H}+\omega^{2} \varepsilon \mu \boldsymbol{H}=0 \quad \text { in } K,
$$

i.e., each component $H_{i}, i=1,2,3$, of $\boldsymbol{H}$ is the solution to the homogeneous Helmholtz equation in $K$ with wavenumber $\kappa:=\omega \sqrt{\varepsilon \mu}$.

Finally, introduce the weighted Sobolev norms

$$
\|v\|_{r, \kappa, K}^{2}=\sum_{j=0}^{r} \kappa^{2(k-j)}|v|_{j, K}^{2} .
$$

The following result is a straightforward application of [38, Lem. 4.5, Cor. 5.5].

Lemma 5.2. Let $K$ be an element of $\mathcal{T}_{h}$ satisfying the assumptions stated in this section, and let $\lambda_{K}$ be the positive parameter depending only on the shape of $K$ introduced in [38, Thm. 3.2], based upon [9. Then, for every $q, r \in \mathbb{N}, q \geq 2 r+1$, $q \geq 2\left(1+2^{1 / \lambda_{K}}\right)$, if $H_{i} \in H^{r+1}(\Omega)$, there exists a set of $p=(q+1)^{2}$ plane wave propagation directions $\left\{\boldsymbol{d}_{\ell}\right\}_{1 \leq \ell \leq p},\left|\boldsymbol{d}_{\ell}\right|=1$, and a corresponding $\vec{\alpha}^{i} \in \mathbb{C}^{p}$ such that, for every $0 \leq j \leq r$,

$$
\begin{aligned}
& \left\|H_{i}-\sum_{1 \leq \ell \leq p} \alpha_{\ell}^{i} e^{i \kappa \boldsymbol{x} \cdot \boldsymbol{d}_{\ell}}\right\|_{j, \kappa, K} \leq C\left(1+\left(\kappa h_{K}\right)^{q+j-r+8}\right) e^{\left(\frac{7}{4}-\frac{3}{4} \rho\right) \kappa h_{K}} h_{K}^{r+1-j} \\
& {\left[q^{-\lambda_{K}(r+1-j)}+(\rho q)^{-\frac{q-3}{2}} M\right]\left\|H_{i}\right\|_{r+1, \kappa, K}}
\end{aligned}
$$

for $i=1,2,3$. Here, the constant $C>0$ only depends on $j, r$ and on the shape of $K, M \leq 2 \sqrt{\pi} p$.

Remark 5.3. If instead of assuming the "optimal" set of directions of [38, Lem. 4.5], we choose the system of directions introduced in 45 and available at the website [49, we have $M \leq 4 \sqrt{\pi} p q$ and the second term in the right-hand side of (5.4) below is multiplied by $q$. The asymptotic bound (5.7) is not affected by this change.

Theorem 5.4. Let $K$ be an element satisfying the assumptions stated in this section. Then, for every $q, r \in \mathbb{N}, q \geq 2 r+1, q \geq 2\left(1+2^{1 / \lambda_{K}}\right)$, with $\lambda_{K}$ as in Lemma [5.2, there exists a set of $p=(q+1)^{2}$ plane wave propagation directions with the corresponding set of polarization directions $d_{2 p}(K)$ defined by (5.1) such that, for every $\boldsymbol{E} \in H^{r+1}($ curl; $K)$ solution of (15.2), there exists $\boldsymbol{\xi}_{\boldsymbol{E}} \in \boldsymbol{V}_{2 p}^{E}(K)$ such that

$$
\begin{array}{r}
\left\|\boldsymbol{E}-\boldsymbol{\xi}_{\boldsymbol{E}}\right\|_{j-1, \kappa, K} \leq C \kappa^{-2}\left(1+\left(\kappa h_{K}\right)^{q+j-r+8}\right) e^{\left(\frac{7}{4}-\frac{3}{4} \rho\right) \kappa h_{K}} h_{K}^{r+1-j} \\
{\left[q^{-\lambda_{K}(r+1-j)}+(\rho q)^{-\frac{q-3}{2}} p\right]\|\nabla \times \boldsymbol{E}\|_{r+1, \kappa, K}}
\end{array}
$$

for every $1 \leq j \leq r$. Here, the constant $C>0$ only depends on $j, r$ and the shape of $K$.

Proof. Given the set of directions $\left\{\boldsymbol{d}_{\ell}\right\}_{1 \leq \ell \leq p}$ of Lemma 5.2, $\boldsymbol{H}$ can be approximated in the space generated by

$$
\left\{(1,0,0) e^{i \kappa \boldsymbol{x} \cdot \boldsymbol{d}_{\ell}},(0,1,0) e^{i \kappa \boldsymbol{x} \cdot \boldsymbol{d}_{\ell}},(0,0,1) e^{i \kappa \boldsymbol{x} \cdot \boldsymbol{d}_{\ell}}\right\}_{1 \leq \ell \leq p}
$$

with the same orders of convergence as in (5.3). 
For every $\ell$, we fix a unit vector $\boldsymbol{a}_{\ell}^{1}$ such that $\left(\boldsymbol{a}_{\ell}^{1}, \boldsymbol{d}_{\ell}\right)_{\mathbb{R}^{3}}=0$; we set $\boldsymbol{a}_{\ell}^{2}=\boldsymbol{a}_{\ell}^{1} \times \boldsymbol{d}_{\ell}$ and $\boldsymbol{a}_{\ell}^{3}=\boldsymbol{d}_{\ell}$. Clearly, $\left\{\boldsymbol{a}_{\ell}^{i}\right\}_{i=1,2,3}$ is an orthonormal basis of $\mathbb{R}^{3}$; therefore, the basis

$$
\left\{\boldsymbol{w}_{\ell}^{i}(\boldsymbol{x}):=\boldsymbol{a}_{\ell}^{i} e^{i \kappa \boldsymbol{x} \cdot \boldsymbol{d}_{\ell}}\right\}_{\substack{1 \leq \ell \leq p \\ i=1,2,3}}
$$

generates the same space as the basis in (5.5). Thus, there exist $\vec{\alpha}^{i} \in \mathbb{C}^{p}, i=1,2,3$ (different from those in (5.3) ), such that, for every $0 \leq j \leq r$,

$$
\begin{aligned}
\left\|\boldsymbol{H}-\sum_{\substack{1 \leq \ell \leq p \\
i=1,2,3}} \alpha_{\ell}^{i} \boldsymbol{w}_{\ell}^{i}\right\|_{j, \kappa, K} \leq & C\left(1+\left(\kappa h_{K}\right)^{q+j-r+8}\right) e^{\left(\frac{7}{4}-\frac{3}{4} \rho\right) \kappa h_{K}} h_{K}^{r+1-j} \\
& {\left[q^{-\lambda_{K}(r+1-j)}+(\rho q)^{-\frac{q-3}{2}} M\right]\|\boldsymbol{H}\|_{r+1, \kappa, K} . }
\end{aligned}
$$

Notice that, while $\boldsymbol{w}_{\ell}^{1}$ and $\boldsymbol{w}_{\ell}^{2}$ are Maxwell-Trefftz functions, this is not true for $\boldsymbol{w}_{\ell}^{3}$; thus, we want to approximate $\boldsymbol{E}$ in the space generated by

$$
\left\{\boldsymbol{w}_{\ell}^{i}(\boldsymbol{x})\right\}_{\substack{1 \leq \ell \leq p \\ i=1,2}} .
$$

On the other hand, simple calculations give

$$
\nabla \times \boldsymbol{w}_{\ell}^{1}=-i \kappa \boldsymbol{w}_{\ell}^{2}, \quad \nabla \times \boldsymbol{w}_{\ell}^{2}=i \kappa \boldsymbol{w}_{\ell}^{1}, \quad \nabla \times \boldsymbol{w}_{\ell}^{3}=0 ;
$$

these identities, together with the Trefftz property of $\boldsymbol{E}$, give (with the same coefficients as in (5.6) ):

$$
\begin{aligned}
& \left\|\boldsymbol{E}-\mu^{1 / 2} \varepsilon^{-1 / 2} \sum_{1 \leq \ell \leq p}\left(-\alpha_{\ell}^{2} \boldsymbol{w}_{\ell}^{1}+\alpha_{\ell}^{1} \boldsymbol{w}_{\ell}^{2}\right)\right\|_{j-1, \kappa, K} \\
& =\left\|\kappa^{-2} \nabla \times \nabla \times \boldsymbol{E}+\mu^{1 / 2} \varepsilon^{-1 / 2}(i \kappa)^{-1} \sum_{\substack{1 \leq \ell \leq p \\
i=1,2,3}} \alpha_{\ell}^{i} \nabla \times \boldsymbol{w}_{\ell}^{i}\right\|_{j-1, \kappa, K} \\
& =\left\|\frac{i}{\omega \varepsilon} \nabla \times\left[(i \omega \mu)^{-1} \nabla \times \boldsymbol{E}-\sum_{\substack{1 \leq \ell \leq p \\
i=1,2,3}} \alpha_{\ell}^{i} \boldsymbol{w}_{\ell}^{i}\right]\right\|_{j-1, \kappa, K} \\
& \leq(\omega \varepsilon)^{-1}\left\|\frac{\nabla \times \boldsymbol{E}}{i \omega \mu}-\sum_{\substack{1 \leq \ell \leq p \\
i=1,2,3}} \alpha_{\ell}^{i} \boldsymbol{w}_{\ell}^{i}\right\|_{j, \kappa, K} \stackrel{\text { def. of } \boldsymbol{H}}{=}(\omega \varepsilon)^{-1}\left\|\boldsymbol{H}-\sum_{\substack{1 \leq \ell \leq p \\
i=1,2,3}} \alpha_{\ell}^{i} \boldsymbol{w}_{\ell}^{i}\right\|_{j, \kappa, K} \\
& \stackrel{\text { (5.6) }}{\leq}(\omega \varepsilon)^{-1} C\left(1+\left(\kappa h_{K}\right)^{q+j-r+8}\right) e^{\left(\frac{7}{4}-\frac{3}{4} \rho\right) \kappa h_{K}} h_{K}^{r+1-j} \\
& {\left[q^{-\lambda_{K}(r+1-j)}+(\rho q)^{-\frac{q-3}{2}} M\right]\|\boldsymbol{H}\|_{r+1, \kappa, K}} \\
& \text { def. of } \boldsymbol{H} \kappa^{-2} C\left(1+\left(\kappa h_{K}\right)^{q+j-r+8}\right) e^{\left(\frac{7}{4}-\frac{3}{4} \rho\right) \kappa h_{K}} h_{K}^{r+1-j} \\
& {\left[q^{-\lambda_{K}(r+1-j)}+(\rho q)^{-\frac{q-3}{2}} M\right]\|\nabla \times \boldsymbol{E}\|_{r+1, \kappa, K} .}
\end{aligned}
$$


Finally, we apply the bound $M \leq 2 \sqrt{\pi} p$ mentioned in Lemma 5.2 and obtain the assertion with

$$
\boldsymbol{\xi}_{E}=\sum_{1 \leq \ell \leq p}\left(-\alpha_{\ell}^{2} \boldsymbol{w}_{\ell}^{1}+\alpha_{\ell}^{1} \boldsymbol{w}_{\ell}^{2}\right)
$$

The following approximation properties in ||$|\cdot| \|_{\mathcal{F}_{h}^{+}}$norm can be derived from Theorem 5.4 by proceeding as in [29, Lem. 3.10, Thm. 3.11].

Corollary 5.5. Assume $\mathcal{T}_{h}$ to satisfy the assumptions stated in this section. Set $\lambda=\min _{K \in \mathcal{T}_{h}} \lambda_{K}$ where $\lambda_{K}$ is defined as in Lemma 5.2. Then, for every $q, r \in \mathbb{N}$, $r \geq 3, q \geq 2 r+1, q \geq 2\left(1+2^{1 / \lambda}\right)$, there exists a set of $p=(q+1)^{2}$ plane wave propagation directions $\left\{\boldsymbol{d}_{\ell}\right\}_{1 \leq \ell \leq p}$ with the corresponding set of polarization directions $d_{2 p}(K)$ defined by (5.1) such that, for every $\boldsymbol{E} \in H^{r+1}\left(\mathrm{curl} ; \mathcal{T}_{h}\right)$ solution of (2.2), there exists $\boldsymbol{\xi}_{h, 2 p} \in \boldsymbol{V}_{2 p}$ such that

$$
\begin{aligned}
\left.\left\|\boldsymbol{E}-\boldsymbol{\xi}_{h, 2 p}\right\|\right|_{\mathcal{F}_{h}^{+}} & \leq C \omega^{-\frac{5}{2}} h^{r-\frac{3}{2}} \Phi(q)\|\nabla \times \boldsymbol{E}\|_{r+1, \omega, \Omega} \\
\Phi(q) & :=\left[q^{-\lambda(r-1)}+(\rho q)^{-\frac{q-3}{2}} p\right]^{\frac{1}{2}}\left[q^{-\lambda(r-2)}+(\rho q)^{-\frac{q-3}{2}} p\right]^{\frac{1}{2}},
\end{aligned}
$$

where $C=C(\omega h)>0$ is independent of $p$ and $\boldsymbol{E}$, but increases as a function of the product $\omega h$ ( $C$ depends on the shape of the elements $K \in \mathcal{T}_{h}, r, \vartheta, \varepsilon, \mu$ and on the flux parameters).

Proof. Since

$$
\|u\|_{0, \partial K}^{2} \leq C\|u\|_{0, K}\left(h_{K}^{-1}\|u\|_{0, K}+|u|_{1, K}\right) \quad \forall u \in H^{1}(K),
$$

with a constant $C>0$ only depending on the shape of $K$ (see [12, Th. 1.6.6]), from Theorem 5.4 there exists $\boldsymbol{\xi}_{h, 2 p} \in \boldsymbol{V}_{2 p}$ such that

$$
\begin{aligned}
\left\|\boldsymbol{E}-\boldsymbol{\xi}_{h, 2 p}\right\|_{0, \partial K}^{2} \leq C e^{\left(\frac{7}{2}-\frac{3}{2} \rho\right) \kappa h_{K}}\left(1+\left(\kappa h_{K}\right)^{2 q-2 r+19}\right) \kappa^{-4} h_{K}^{2 r-1} \\
\cdot\left[q^{-\lambda r}+(\rho q)^{-\frac{q-3}{2}} p\right]\left[q^{-\lambda(r-1)}+(\rho q)^{-\frac{q-3}{2}} p\right]\|\nabla \times \boldsymbol{E}\|_{r+1, \kappa, K}^{2}
\end{aligned}
$$

and

$$
\begin{aligned}
\left\|\nabla \times\left(\boldsymbol{E}-\boldsymbol{\xi}_{h, 2 p}\right)\right\|_{0, \partial K}^{2} \leq C e^{\left(\frac{7}{2}-\frac{3}{2} \rho\right) \kappa h_{K}}\left(1+\left(\kappa h_{K}\right)^{2 q-2 r+21}\right) \kappa^{-4} h_{K}^{2 r-3} \\
\cdot\left[q^{-\lambda(r-1)}+(\rho q)^{-\frac{q-3}{2}} p\right]\left[q^{-\lambda(r-2)}+(\rho q)^{-\frac{q-3}{2}} p\right]\|\nabla \times \boldsymbol{E}\|_{r+1, \kappa, K}^{2}
\end{aligned}
$$

for all $K \in \mathcal{T}_{h}$, with $C>0$ only depending on the shape of $K$ and on $r$. Thus,

$$
\begin{aligned}
\omega\left\|\boldsymbol{E}-\boldsymbol{\xi}_{h, 2 p}\right\|_{0, \mathcal{F}_{h}}^{2} \leq C e^{\left(\frac{7}{2}-\frac{3}{2} \rho\right) \kappa h}\left(1+(\kappa h)^{2 q-2 r+19}\right) \kappa^{-3} h^{2 r-1} \\
\cdot\left[q^{-\lambda r}+(\rho q)^{-\frac{q-3}{2}} p\right]\left[q^{-\lambda(r-1)}+(\rho q)^{-\frac{q-3}{2}} p\right]\|\nabla \times \boldsymbol{E}\|_{r+1, \kappa, \Omega}^{2}
\end{aligned}
$$

and

$$
\begin{aligned}
\omega^{-1} \| \nabla \times & \left(\boldsymbol{E}-\boldsymbol{\xi}_{h, 2 p}\right) \|_{0, \mathcal{F}_{h}}^{2} \leq C e^{\left(\frac{7}{2}-\frac{3}{2} \rho\right) \kappa h}\left(1+(\kappa h)^{2 q-2 r+21}\right) \kappa^{-5} h^{2 r-3} \\
& \cdot\left[q^{-\lambda(r-1)}+(\rho q)^{-\frac{q-3}{2}} p\right]\left[q^{-\lambda(r-2)}+(\rho q)^{-\frac{q-3}{2}} p\right]\|\nabla \times \boldsymbol{E}\|_{r+1, \kappa, \Omega}^{2}
\end{aligned}
$$


with $C>0$ only depending on the shape of the elements $K \in \mathcal{T}_{h}$ and on $r$. This, together with the definition of $\||\cdot|\|_{\mathcal{F}_{h}^{+}}$, gives

$$
\begin{aligned}
\left\|\boldsymbol{E}-\boldsymbol{\xi}_{h, 2 p} \mid\right\|_{\mathcal{F}_{h}^{+}} \leq C e^{\left(\frac{7}{4}-\frac{3}{4} \rho\right) \kappa h}(\kappa h)^{-\frac{5}{2}}\left(1+(\kappa h)^{q-r+\frac{21}{2}}\right) h^{r+1} \\
\cdot\left[q^{-\lambda(r-1)}+(\rho q)^{-\frac{q-3}{2}} p\right]^{\frac{1}{2}}\left[q^{-\lambda(r-2)}+(\rho q)^{-\frac{q-3}{2}} p\right]^{\frac{1}{2}}\|\nabla \times \boldsymbol{E}\|_{r+1, \kappa, \Omega},
\end{aligned}
$$

with $C>0$ only depending on the shape of the elements $K \in \mathcal{T}_{h}, r, \vartheta, \varepsilon, \mu$, and on the flux parameters (but independent of $\boldsymbol{E}, h, p$ and $\omega$ ), from which the result directly follows.

Remark 5.6. Asymptotically, $\Phi(q)$ behaves, for increasing $q$, as $q^{-\lambda\left(r-\frac{3}{2}\right)}$. Therefore, for large $q$, the estimate of Corollary 5.5 can be written as

$$
\left\|\boldsymbol{E}-\boldsymbol{\xi}_{h, 2 p}\left|\left\|\left.\right|_{\mathcal{F}_{h}^{+}} \leq C \omega^{-\frac{5}{2}}\left(\frac{h}{q^{\lambda}}\right)^{r-\frac{3}{2}}\right\| \nabla \times \boldsymbol{E} \|_{r+1, \omega, \Omega} .\right.\right.
$$

5.2. Convergence rates. Inserting the estimates (5.7) within Theorem 4.3 and Theorem 4.9, we have the following convergence rates.

Theorem 5.7. Assume that the analytical solution $\boldsymbol{E}$ to the Maxwell problem (2.2) belongs to $H^{r+1}(\operatorname{curl} ; \Omega), r \in \mathbb{N}, r \geq 3$. Assume that the mesh $\mathcal{T}_{h}$ satisfies the assumptions stated in Section [5.1, set $\lambda=\min _{K \in \mathcal{T}_{h}} \lambda_{K}$ where $\lambda_{K}$ is defined as in Lemma 5.2, and let $\boldsymbol{E}_{h, p} \in \boldsymbol{V}_{2 p}\left(\mathcal{T}_{h}\right), p=(q+1)^{2} \in \mathbb{N}$, with $q \geq 2 r+1$, $q \geq 2\left(1+2^{1 / \lambda}\right)$, be the $P W D G$ numerical solution.

Then, there exist two constants $C_{1}, C_{2}>0$ independent of $p$ but depending on $\omega$ and $h$ only through the product $\omega h$ as an increasing function, such that, for large $p$,

$$
\begin{gathered}
\left.\left\|\boldsymbol{E}-\boldsymbol{E}_{h, p}\right\|\right|_{\mathcal{F}_{h}} \leq C_{1} \omega^{-\frac{5}{2}}\left(\frac{h}{q^{\lambda}}\right)^{r-\frac{3}{2}}\|\nabla \times \boldsymbol{E}\|_{r+1, \omega, \Omega}, \\
\left\|\boldsymbol{E}-\boldsymbol{E}_{h, p}\right\|_{H(\mathrm{div} ; \Omega)^{\prime}} \leq C_{2}\left(\omega^{-\frac{5}{2}}+\omega^{-4}\right) \frac{h^{r-2}}{q^{\lambda\left(r-\frac{3}{2}\right)}}\|\nabla \times \boldsymbol{E}\|_{r+1, \omega, \Omega} .
\end{gathered}
$$

Here, $C_{1}=C_{1}(\omega h)$ and $C_{2}=C_{2}(\omega h)$ depend on the shape of the elements $K \in \mathcal{T}_{h}$, $r, \vartheta, \varepsilon, \mu$, and on the flux parameters; $C_{2}$ also depends on $\Omega$, s.r. $\left(\mathcal{T}_{h}\right)$, and q.u. $\left(\mathcal{T}_{h}\right)$.

Proof. The first bound is straightforward. To derive the second bound, we simply notice that, for $f(\omega, h)$ defined by (4.6) we have

$$
f(\omega, h) \leq C h^{-\frac{1}{2}}\left(1+\omega^{-\frac{3}{2}}\right),
$$

where $C>0$ only depends on $\Omega$ and on the product $\omega h$ as an increasing function.

Remark 5.8. For functions which possess analytic extensions outside $K$, the convergence rates of the error bound in Lemma 5.2 are exponential in $p$, as explained in [38, Rem. 3.3] and [29, Rem. 3.14]. Therefore, if $\boldsymbol{E}$ admits an analytic extension outside $\Omega$, the convergence of the estimates in Corollary 5.5, Remark [5.6] and thus in Theorem 5.7 is exponential in $p$ (see [29, Rem. 3.14] and [29, Sec. 4] for numerical evidence in the Helmholtz case). 


\section{Conclusions}

We have extended to the time-harmonic Maxwell problem the $p$-version error analysis framework developed in [29] for Trefftz-DG methods for the Helmholtz equation.

Due to the assumptions on the regularity of the solution required in the duality argument, our analysis is restricted to the case of globally constant material coefficients, even though the formulation of the Trefftz-DG methods allows for piecewise constant coefficients. The obtained error estimates are not in the $L^{2}-$ norm, as for the Helmholtz problem, but in a weaker norm which, on the other hand, coincides with the $L^{2}$-norm for the exact fields.

The presented analysis covers general Trefftz-DG methods, independently of the particular choice of the local approximation spaces. Possible choices are vector spherical waves, fundamental solutions, plane waves, or combinations of them; see, for instance, [8]. Here, for particular plane wave approximation spaces, we have also derived convergence rates of the corresponding Trefftz-DG method. These rates might be improved with a more sophisticated best approximation analysis; in this context, extensions of Vekua's theory to the Maxwell setting could be worth exploring.

\section{REFERENCES}

1. M. Ainsworth, Discrete dispersion relation for $h p$-version finite element approximation at high wave number, SIAM J. Numer. Anal. 42 (2004), no. 2, 563-575. MR2084226 (2005i:65174)

2. Dispersive and dissipative behaviour of high order discontinuous Galerkin finite element methods, J. Comput. Phys. 198 (2004), no. 1, 106-130. MR2071391 (2005d:65161)

3. __ Dispersive properties of high order Nédélec/edge element approximation of the timeharmonic Maxwell equations, Philos. Trans. R. Soc. Lond. Ser. A Math. Phys. Eng. Sci. 362 (2004), no. 1816, 471-491. MR2075903

4. M. Amara, R. Djellouli, and C. Farhat, Convergence analysis of a discontinuous Galerkin method with plane waves and Lagrange multipliers for the solution of Helmholtz problems, SIAM J. Numer. Anal. 47 (2009), no. 2, 1038-1066. MR2485444 (2010a:65226)

5. C. Amrouche, C. Bernardi, M. Dauge, and V. Girault, Vector potentials in three-dimensional non-smooth domains, Math. Methods Appl. Sci. 21 (1998), no. 9, 823-864. MR.1626990 (99e:35037)

6. D. N. Arnold, F. Brezzi, B. Cockburn, and L. D. Marini, Unified analysis of discontinuous Galerkin methods for elliptic problems, SIAM J. Numer. Anal. 39 (2002), no. 5, 1749-1779. MR.1885715 (2002k:65183)

7. I. Babuška and J. M. Melenk, The partition of unity method, Internat. J. Numer. Methods Engrg. 40 (1997), no. 4, 727-758. MR1429534(97j:73071)

8. Z. Badics and Y. Matsumoto, Trefftz discontinuous Galerkin methods for time-harmonic electromagnetic and ultrasound transmission problems, International Journal of Applied Electromagnetics and Mechanics 28 (2008), no. 1-2, 17-24.

9. T. Bagby, L. Bos, and N. Levenberg, Quantitative approximation theorems for elliptic operators, J. Approx. Theory 85 (1996), no. 1, 69-87. MR1382051 (97h:41060)

10. S. C. Brenner, F. Li, and L.-Y. Sung, A locally divergence-free nonconforming finite element method for the reduced time-harmonic Maxwell equation, Math. Comput. 76 (2007), 573-595. MR2291828 (2008c:65316)

11. S. C. Brenner, F. Li, and L.-Y. Sung, Nonconforming Maxwell eigensolvers, J. Sci. Comput. 40 (2009), no. 1-3, 51-85. MR2511728 (2010i:65251)

12. S. C. Brenner and L. R. Scott, Mathematical theory of finite element methods, 3rd ed., Texts Appl. Math., Springer-Verlag, New York, 2007. 
13. A. Buffa and P. Ciarlet, Jr., On traces for functional spaces related to Maxwell's equations. I. An integration by parts formula in Lipschitz polyhedra, Math. Methods Appl. Sci. 24 (2001), no. 1, 9-30. MR.1809491 (2002b:78024)

14. A. Buffa, M. Costabel, and C. Schwab, Boundary element methods for Maxwell's equations on non-smooth domains, Numer. Math. 92 (2002), no. 4, 679-710. MR.1935806(2003k:65159)

15. A. Buffa and P. Monk, Error estimates for the ultra weak variational formulation of the Helmholtz equation, M2AN, Math. Model. Numer. Anal. 42 (2008), no. 6, 925-940. MR2473314(2009j:65295)

16. A. Buffa and I. Perugia, Discontinuous Galerkin approximation of the Maxwell eigenproblem, SIAM J. Numer. Anal. 44 (2006), no. 5, 2198-2226. MR.2263045 (2007k:65173)

17. O. Cessenat, Application d'une nouvelle formulation variationnelle aux équations d'ondes harmoniques, Problèmes de Helmholtz $2 D$ et de Maxwell 3D, Ph.D. thesis, Université Paris IX Dauphine, 1996.

18. O. Cessenat and B. Després, Application of an ultra weak variational formulation of elliptic PDEs to the two-dimensional Helmholtz equation, SIAM J. Numer. Anal. 35 (1998), no. 1, 255-299. MR1618464 (99b:65139)

19. _ Using plane waves as base functions for solving time harmonic equations with the ultra weak variational formulation, J. Comput. Acoust. 11 (2003), 227-238. MR2013687 (2004j:65182)

20. D. Copeland, U. Langer, and D. Pusch, From the boundary element domain decomposition methods to local Trefftz finite element methods on polyhedral meshes., Bercovier, Michel (ed.) et al., Domain decomposition methods in science and engineering XVIII. Berlin: Springer. Lecture Notes in Computational Science and Engineering 70, 315-322, 2009. MR2762421 (2011m:65299)

21. E. Creusé and S. Nicaise, Discrete compactness for a discontinuous Galerkin approximation of Maxwell's system, M2AN Math. Model. Numer. Anal. 40 (2006), 413-430. MR2241830 (2008a:65207)

22. G. Gabard, Discontinuous Galerkin methods with plane waves for time-harmonic problems, J. Comput. Phys. 225 (2007), 1961-1984. MR2349691 (2009e:65173)

23. G. Gabard, P. Gamallo, and T. Huttunen, A comparison of wave-based discontinuous Galerkin, ultra-weak and least-square methods for wave problems, Internat. J. Numer. Methods Engrg. 85 (2011), no. 3, 380-402. MR2779291(2011i:65211)

24. C. J. Gittelson, R. Hiptmair, and I. Perugia, Plane wave discontinuous Galerkin methods: analysis of the h-version, M2AN Math. Model. Numer. Anal. 43 (2009), no. 2, 297-332. MR2512498 (2010b:65255)

25. P. Grisvard, Elliptic problems in nonsmooth domains, Monogr. Stud. Math., vol. 24, Pitman, Boston, 1985. MR775683 (86m:35044)

26. _ـ Singularities in boundary value problems, Recherches en Mathématiques Appliquées [Research in Applied Mathematics], vol. 22, Masson, Paris, 1992. MR1173209 (93h:35004)

27. J. S. Hesthaven and T. Warburton, High-order nodal discontinuous Galerkin methods for the Maxwell eigenvalue problem, Philos. Trans. R. Soc. Lond. Ser. A Math. Phys. Eng. Sci. 362 (2004), no. 1816, 493-524. MR2075904

28. R. Hiptmair, A. Moiola, and I. Perugia, Stability results for the time-harmonic Maxwell equations with impedance boundary conditions, Math. Mod. Meth. Appl. Sci. 21 (2011), 22632287. MR 2860676

29. _ Plane wave discontinuous Galerkin methods for the 2D Helmholtz equation: analysis of the p-version, SIAM J. Numer. Anal. 49 (2011), 264-284. MR2783225

30. R. Hiptmair and I. Perugia, Mixed plane wave discontinuous Galerkin methods, Domain Decomposition Methods in Science and Engineering XVIII (M. Bercovier, M. J. Gander, R. Kornhuber, and O. Widlund, eds.), Lect. Notes Comput. Sci. Eng., Springer, 2008, pp. 51-62. MR2743958 (2012b:65182)

31. P. Houston, I. Perugia, A. Schneebeli, and D. Schötzau, Interior penalty method for the indefinite time-harmonic Maxwell equations, Numer. Math. 100 (2005), no. 3, 485-518. MR2194528 (2006k:65323)

32. P. Houston, I. Perugia, A. Schneebeli, and D. Schötzau, Mixed discontinuous Galerkin approximation of the Maxwell operator: the indefinite case, M2AN Math. Model. Numer. Anal. 39 (2005), no. 4, 727-753. MR2165677(2006h:65186) 
33. P. Houston, I. Perugia, and D. Schötzau, hp-DGFEM for Maxwell's equations, Numerical mathematics and advanced applications, Springer Italia, Milan, 2003, pp. 785-794. MR2360853 (2008i:78011)

34. T. Huttunen, M. Malinen, and P. Monk, Solving Maxwell's equations using the ultra weak variational formulation, J. Comput. Phys. 223 (2007), 731-758. MR2319231(2008b:65133)

35. T. Huttunen and P. Monk, The use of plane waves to approximate wave propagation in anisotropic media, J. Comput. Math. 25 (2007), no. 3, 350-367. MR2320239 (2009b:78012)

36. T. Huttunen, P. Monk, and J. P. Kaipio, Computational aspects of the ultra-weak variational formulation, J. Comput. Phys. 182 (2002), no. 1, 27-46. MR.1936802 (2003j:65107)

37. J. M. Melenk, On approximation in meshless methods, Frontiers of numerical analysis, Universitext, Springer, Berlin, 2005, pp. 65-141. MR2178270(2006h:65191)

38. A. Moiola, R. Hiptmair, and I. Perugia, Plane wave approximation of homogeneous Helmholtz solutions, Z. Angew. Math. Phys. 62 (2011), no. 5, 809-837.

39. _ Vekua theory for the Helmholtz operator, Z. Angew. Math. Phys. 62 (2011), no. 5, 779-807.

40. P. Monk, Finite element methods for Maxwell's equations, Numerical Mathematics and Scientific Computation, Oxford University Press, 2003. MR2059447 (2005d:65003)

41. P. Monk and D.Q. Wang, A least squares method for the Helmholtz equation, Comput. Methods Appl. Mech. Eng. 175 (1999), no. 1/2, 121-136. MR1692914(2000c:76051)

42. I. Perugia and D. Schötzau, The hp-local discontinuous Galerkin method for low-frequency time-harmonic Maxwell's equations, Math. Comput. 72 (2003), 1179-1214. MR.1972732 (2004b:65190)

43. I. Perugia, D. Schötzau, and P. Monk, Stabilized interior penalty methods for the timeharmonic Maxwell equations, Comput. Methods Appl. Mech. Eng. 191 (2002), 4675-4697. MR.1929626 (2003j:78058)

44. H. Riou, P. Ladevéze, and B. Sourcis, The multiscale VTCR approach applied to acoustics problems, J. Comput. Acoust. 16 (2008), no. 4, 487-505.

45. I. H. Sloan and R. S. Womersley, Extremal systems of points and numerical integration on the sphere, Adv. Comput. Math. 21 (2004), no. 1-2, 107-125. MR2065291 (2005b:65024)

46. R. Tezaur and C. Farhat, Three-dimensional discontinuous Galerkin elements with plane waves and Lagrange multipliers for the solution of mid-frequency Helmholtz problems, Internat. J. Numer. Methods Engrg. 66 (2006), no. 5, 796-815. MR2219901 (2007a:65215)

47. I. Tsukerman, Electromagnetic applications of a new finite-difference calculus, IEEE Transactions on Magnetics 41 (2005), no. 7, 2206-2225.

48. T. Warburton and M. Embree, The role of the penalty in the local discontinuous Galerkin method for Maxwell's eigenvalue problem, Comput. Methods Appl. Mech. Engrg. 195 (2006), no. 25-28, 3205-3223. MR2220916 (2007e:65125)

49. R. S. Womersley and I. H. Sloan, Interpolation and cubature on the sphere, http://web. maths. unsw.edu. au/ ${ }^{\text {rsw/Sphere/. }}$

Seminar for Applied Mathematics, ETH Zürich, 8092 Zürich, Switzerland

E-mail address: ralf.hiptmair@sam.math.ethz.ch

Seminar for Applied Mathematics, ETH Zürich, 8092 Zürich, Switzerland

E-mail address: andrea.moiola@sam.math.ethz.ch

Current address: Department of Mathematics and Statistics, University of Reading,

Whiteknights, P.O. Box 220, Reading RG6 6AX, UK

Dipartimento di Matematica, Università di Pavia, 27100 Pavia, Italy

E-mail address: ilaria.perugia@unipv.it 